\title{
A mixed POD-PGD approach to parametric thermal urban soil modeling: Application to canyon streets
}

\author{
AZAM Marie-Hélène ${ }^{\mathrm{a}, \mathrm{b}}$, GUERNOUTI Sihem ${ }^{\mathrm{a}, \mathrm{b}, *}$, MUSY Marjorie ${ }^{\mathrm{a}, \mathrm{b}}$, BERGER Julien ${ }^{\mathrm{c}}$, \\ POULLAIN Philippe ${ }^{\mathrm{b}}$, RODLER Auline ${ }^{\mathrm{d}}$ \\ Nantes, FRANCE \\ ${ }^{a}$ Cerema, F-44000 Nantes, France \\ ${ }^{b}$ Univ. Nantes, CNRS UMR 6183, GeM, F-44000 Nantes, France \\ ${ }^{c}$ Univ. Grenoble Alpes, Univ. Savoie Mont Blanc, UMR 5271 CNRS, LOCIE, F-73000 Chambéry, France \\ ${ }^{d}$ Ensa Nantes, CRENAU UMR CNRS/ECN/MCC 1563 F-44200 Nantes, France
}

\footnotetext{
*Corresponding author. E-mail address: sihem.guernouti@cerema.fr Address: Cerema, 9 rue René Viviani, 44000 Nantes, France
} 


\title{
A mixed POD-PGD approach to parametric thermal urban soil modeling:
}

\section{Application to canyon streets}

\begin{abstract}
Numerical simulation is a powerful tool for assessing the causes of an Urban Heat Island (UHI) effect or quantifying the impact of mitigation solutions on local climatic conditions. However, the numerical cost associated with such a tool, which may seem low for a section of mesh within the district geometric model, is quite significant at the scale of an entire district. Today, the main challenge consists of achieving both a proper representation of the physical phenomena and a critical reduction in the numerical costs of running simulations. This paper presents a combined parametric urban soil model that accurately reproduces thermal heat flux exchanges between the soil and the urban environment with a reduced computation time. For this purpose, the use of a combination of two reduced-order methods is proposed herein: the Proper Orthogonal Decomposition (POD) method, and the Proper Generalized Decomposition (PGD) method. The developed model is applied to two case studies in order to establish a practical evaluation: an open area independent of the influences of the surrounding surface, namely a parking lot, and a theoretical urban scene with two canyon streets. The mean surface temperature reduction error remains below $0.52^{\circ} \mathrm{C}$ for a cut computational cost of $80 \%$.
\end{abstract}

\section{Keyword}

POD PGD parametric model Heat transfer Urban soil model SOLENE-microclimat Urban Heat Island Model Order Reduction

\section{Highlights}

- We propose a parametric model dedicated to urban soil thermal modeling.

- A combination of two reduced-order methods, i.e. POD and PGD, is presented.

- Calculated temperatures are evaluated with respect to in situ measurements.

- The parametric soil model is coupled with the SOLENE-microclimat tool. 
- Its accuracy and computational cost are evaluated in an urban setting. 


\section{Contents}

1 Introduction $\quad 6$

2 Methods $\quad 9$

2.1 Physical problem and Large Original Model (LOM) . . . . . . . . . . . . . . 9

2.2 Building the combined parametric model . . . . . . . . . . . . . . . . . 10

2.2.1 Use of the POD to parameterize the initial condition . . . . . . . . 12

2.2.2 Building the PGD parametric solution . . . . . . . . . . . . . . 13

2.3 Implementation methodology . . . . . . . . . . . . . . . . . . . . . 17

2.4 Assessment methodology . . . . . . . . . . . . . . . . . . . . . . . . . . . 19

$\begin{array}{llr}3 & \text { Results } & 20\end{array}$

3.1 Evaluation of the numerical method with an analytical solution . . . . . . 20

3.2 Application to a case study independent of the influence of the urban environ-

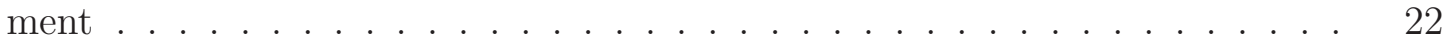

3.2.1 Presentation of the measurement campaign . . . . . . . . . . . . 22

3.2.2 Combined parametric model set-up . . . . . . . . . . . . . . . . . 22

3.2.3 Combined parametric model assessment . . . . . . . . . . . . . . . . 24

3.2.4 Influence the learning period . . . . . . . . . . . . . . . . . 28

3.3 Practical application to an urban environment . . . . . . . . . . . . . . 30

3.3.1 Description of the urban scene . . . . . . . . . . . . . . . . . 30

3.3.2 Combined parametric model setup . . . . . . . . . . . . . . . . 33

3.3.3 Coupling method . . . . . . . . . . . . . . . . . . . . 33

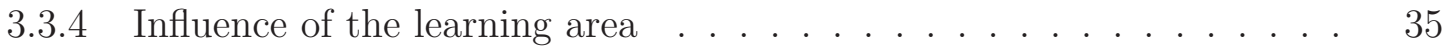

3.3.5 Calculation cost and time . . . . . . . . . . . . . . 38

4 Conclusion $\quad 39$

A Description of the analytical solution $\quad 41$

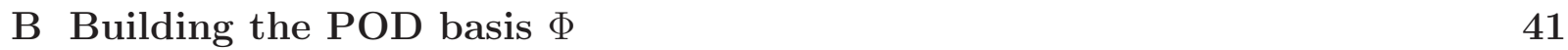


C.1 Computation of $F_{m}^{p}\left(T^{n}\right)$ from $X_{m}^{p}(x)$ and $G_{m}^{p-1}(\psi) \ldots \ldots \ldots \ldots$

C.2 Computation of $G_{m}^{p}(\psi)$ from $X_{m}^{p}(x)$ and $F_{m}^{p}\left(T^{n}\right) \ldots \ldots \ldots . \ldots . \ldots$ 


\begin{tabular}{|c|c|c|c|}
\hline \multicolumn{4}{|c|}{ Nomenclature } \\
\hline$\alpha$ & Thermal diffusivity of the soil $\left[\mathrm{m}^{2} \mathrm{~s}^{-1}\right]$ & $k$ & Thermal conductivity $\left[W \cdot m^{-1} \cdot K^{-1}\right]$ \\
\hline$\gamma$ & Auto-correlation matrix & $Q$ & Snapshots matrix \\
\hline$\lambda_{i}$ & Eigenvalue $i$ & $q_{\ell}$ & Latent heat flux $\left[W \cdot m^{-2}\right]$ \\
\hline$\lambda$ & Eigenvalues diagonal matrix & $q_{c}$ & Sensible heat flux $\left[W \cdot m^{-2}\right]$ \\
\hline $\mathcal{N}$ & Reduction order of the POD basis & $q_{n e t}$ & Net radiative heat flux $\left[W \cdot m^{-2}\right]$ \\
\hline $\begin{array}{l}\Phi \\
\tilde{T}(x, t)\end{array}$ & $\begin{array}{l}\text { POD Reduced order basis } \\
\text { Approximation of the temperature profile }\end{array}$ & $q_{w}$ & Runoff convective heat flux $\left[W \cdot m^{-2}\right]$ \\
\hline$\zeta$ & Unknown of the POD reduced model & $T(x, t)$ & Temperature profile \\
\hline$c$ & Specific Heat Capacity $\left[J \cdot m^{-3} \cdot K^{-1}\right]$ & $T_{\infty}$ & Temperature of the soil below a meter $[K$ \\
\hline$h$ & $\begin{array}{l}\text { Convective heat transfer coefficient } \\
{\left[W \cdot m^{-2} \cdot K^{-1}\right]}\end{array}$ & $\begin{array}{l}T_{a}(t) \\
\mathrm{L}\end{array}$ & $\begin{array}{l}\text { Air temperature }[K] \\
\text { width of the soil layer }\end{array}$ \\
\hline
\end{tabular}

\section{Introduction}

Curbing urban sprawl is a key current objective for urban planners. In the context of global warming, urban densification has negative consequences on local city climate; specifically, it leads to intensification of the urban heat island (UHI) phenomenon [1-3]. For this reason, cities must now be developed in considering UHI as an environmental challenge since it has several consequences on both outdoor comfort and building energy consumption, which can lead to serious health issues.

The storage of solar heat flux by urban materials during the day and its release at night is a main cause of UHI. This heat flux is greater in urban areas than in rural parts due to the higher inertia of construction materials. Surface temperatures drive the various heat fluxes (convection, conduction and long-wave radiative exchanges) at the urban surface. As such, it is of major importance to assess these variables of the urban micro-climate.

In order to mitigate the UHI, its causes must be fully understood and the impact of mitigation solutions on the local micro-climate needs to be quantified. For this purpose, numerical simulation proves to be a powerful tool. Several models have been developed to simulate UHI and its consequences; these include TEB [4] or ARPS-VUC [5], at the city scale, 


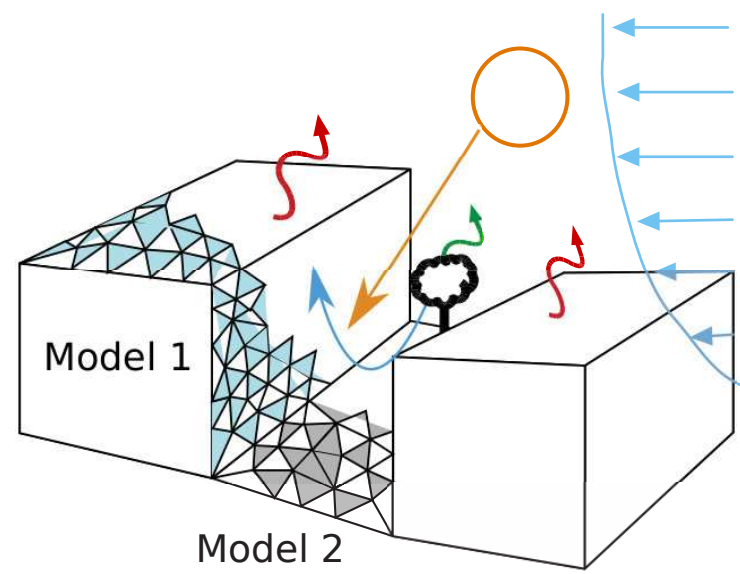

(a) Thermo-radiative balance and mesh represenprocess.

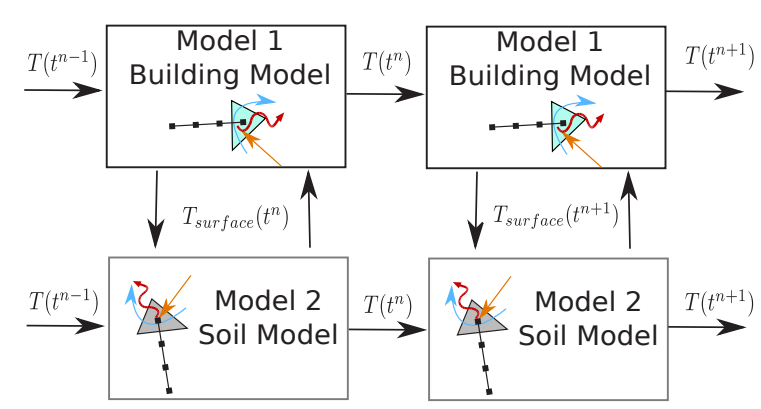

(b) Co-simulation tation

Figure 1: Thermo-radiative balance of the urban scene with co-simulation

Most of these micro-climate tools cited combine several one-dimensional models (soil, buildings, etc.) using a co-simulation approach. The urban scene is meshed as presented in Figure 1; moreover, for each piece of mesh, the thermo-radiative balance of an urban scene is derived. This energy balance is composed of both the short- and long-wave radiative heat fluxes, as well as the sensible and latent heat fluxes. At each time step $t^{n}$, all models exchange parameters like surface temperature and net radiative heat flux balance with the other models. During the interval $t \in\left[t^{n}, t^{n+1}\right]$, each model computes the field of interest, which consists of the temperatures of each surface. Figure 1 displays the co-simulation

(1)

In this context, an initial study has been conducted to improve SOLENE-microclimat soil model performance [10]. A previous study had focused on the ability of the model to reproduce the particular physical phenomenon. The influence of a large number of parameters has been assessed in order to improve the model; these include: material properties, layer size, depth boundary conditions, convective heat transfer coefficient, and discretization. These improvements however have increased the model computation time. Indeed, at each time step, the heat transfer equation in the soil must be solved for each spatial mesh of the urban scene. All told, at each time step, thousands of model computations are processed, thus 
significantly increasing the computation time. The numerical cost, which may seem small for a portion of the mesh, is considerable at the scale of thousands of such portions. Since our requirements have been raised to an extent commensurate with improved computational resources, the main challenge today remains combining the best representation of the physical phenomena with a critical reduction in associated numerical costs.

In this same aim, Gasparin et al. (2017, [11]) proposed combining an analytical approach with a finite difference approach in order to compute the temperature and moisture fields in porous soils. The analytical solution is implemented in the deep soil while the numerical scheme is used to solve the upper layer, where the variations are more pronounced. The two methods are linked through an iterative procedure. For our specific case [11], they were able to reduce computational cost by $16 \%$ to $12 \%$, respectively. This method however is counterproductive since it requires an iterative procedure at each time step.

Our objective herein is to propose a reduced-order model (ROM) for the soil heat transfer equation. This model needs to accurately reproduce the heat flux being exchanged between the soil and its environment for several weeks in offering reduced computation times.

As described above, the thermal modeling of the specific urban soil depends on several parameters, i.e. the thermal characteristics of the soil, the initial temperature profile, the surface energy balance, and the temperature deep in the soil. The thermal characteristics could be considered as a constant over time, though the other parameters evolve at each time step. The transient heat equation thus needs to be solved at each time step. In order to reduce computation time, a parametric model is being proposed. For this purpose, the Proper Generalized Decomposition (PGD) method has been selected. The PGD is an a priori model order-reduction method based on distinct variables representing multidimensional problems. This property allows us to circumvent the "curse of dimensionality". Any variable (i.e. initial or boundary condition) can then be defined as an extra-parameter of a model. Hence, the model solution is given as a direct function of $x$ and $t$ coordinates, along with the extraparameter of the problem. Various examples using the PGD parametric model can be found in applications to heat transfer [12-14] with an emphasis on building energy simulation [14$17]$.

As presented in several references $[18,19]$ devoted to parametric PGD models, when a 
dynamic problem needs to be parameterized, the initial conditions must be taken as a problem parameter; this step increases the number of required model parameters. In order to reduce this overall number of parameters, the initial condition should be parameterized. As proposed by Gonzalez et al. (2014, [20]) and Cueto et al. (2016, [18]), the Proper Orthogonal Decomposition (POD) method is used to parameterize the initial condition, making it an a posteriori model order-reduction method. Consequently, a mixed reduction method approach, based on both POD and PGD, is being proposed to generate a combined parametric soil model in the context of a micro-climate simulation.

The remainder of this paper is organized as follows. The mixed approach is presented step-by-step in Section 2 and then validated using an analytical solution in Section 3.1. The combined parametric soil model is applied to a case study involving a parking lot in Section 3.2. Next, the thermal response of this soil model is compared to in situ measurements in order to evaluate its performance. Once validated under actual boundary conditions, the combined parametric model is applied to an urban scene in Section 3.3. For this purpose, it is coupled with the SOLENE-microclimat tool. Lastly, since the main goal of our work is to reduce computation time, the CPU times of the combined POD+PGD and finite difference models, in conjunction with the SOLENE-microclimat tool, are compared.

\section{Methods}

\subsection{Physical problem and Large Original Model (LOM)}

This model is being dedicated to urban pavement surfaces, such as parking lots and sidewalks. Since such surfaces are considered to be impervious, only heat transfer will be taken into account (i.e. water transfer will be neglected). The physical problem thus involves transient one-dimensional heat conduction through a soil column (Eq. 1) for time interval $\Omega_{t}=[0, \tau]$ and space interval $\Omega_{x}=[0, L]:$

$$
c \frac{\partial T}{\partial t}=\frac{\partial}{\partial x}\left(k \frac{\partial T}{\partial x}\right)
$$

where $k$ is the thermal conductivity and $c$ the specific heat capacity. Both these variables 
depend on the space variable $x$ since the domain, i.e. the soil, is composed of several layers.

These thermal properties are assumed to remain constant over time. At the surface $x=0$, a RoBIN boundary condition is assumed:

$$
-k \frac{\partial T}{\partial x}=q_{n e t}-q_{\ell}-q_{w}-h\left(T(x=0)-T_{a}\right),
$$

西

where $q_{n e t}$ is the net radiative heat flux, $q_{\ell}$ the latent heat flux and $q_{w}$ the runoff convective heat flux. The two last heat fluxes are only computed under rainfall or watering events. The sensible heat flux is calculated from air temperature $T_{a}$ varying over time and from a convective heat transfer coefficient $h$. At the bottom $x=L$, the soil temperature is imposed through DiRICHLET boundary conditions:

$$
T=T_{\infty}
$$

where $T_{\infty}$ is a constant daily temperature [10]. At $t=0$, the initial temperature is set using a space temperature profile:

$$
T=T_{0}(x)
$$

The problem in Equation (1) can be solved using any classical numerical method. The so-called Large Original Model (LOM) is defined as the solution to the previous problem with the finite difference method. The spatial domain $\Omega_{x}$ is discretized into a grid composed of $\mathrm{Nx}$ nodes, while the time domain $\Omega_{t}$ is discretized into $\mathrm{Nt}$ time steps. An implicit scheme is employed, with backward first-order derivatives for the time derivation and a center secondorder derivative scheme for the spatial derivation. In the following sections, the construction of this combined parametric reduced-order models (ROM) for Eq. (1) will be detailed.

\subsection{Building the combined parametric model}

The PGD method is used in order to propose an accurate parametric solution of the formulated soil problem with a shorter computation time, in anticipation of coupling with a micro-climate tool. 


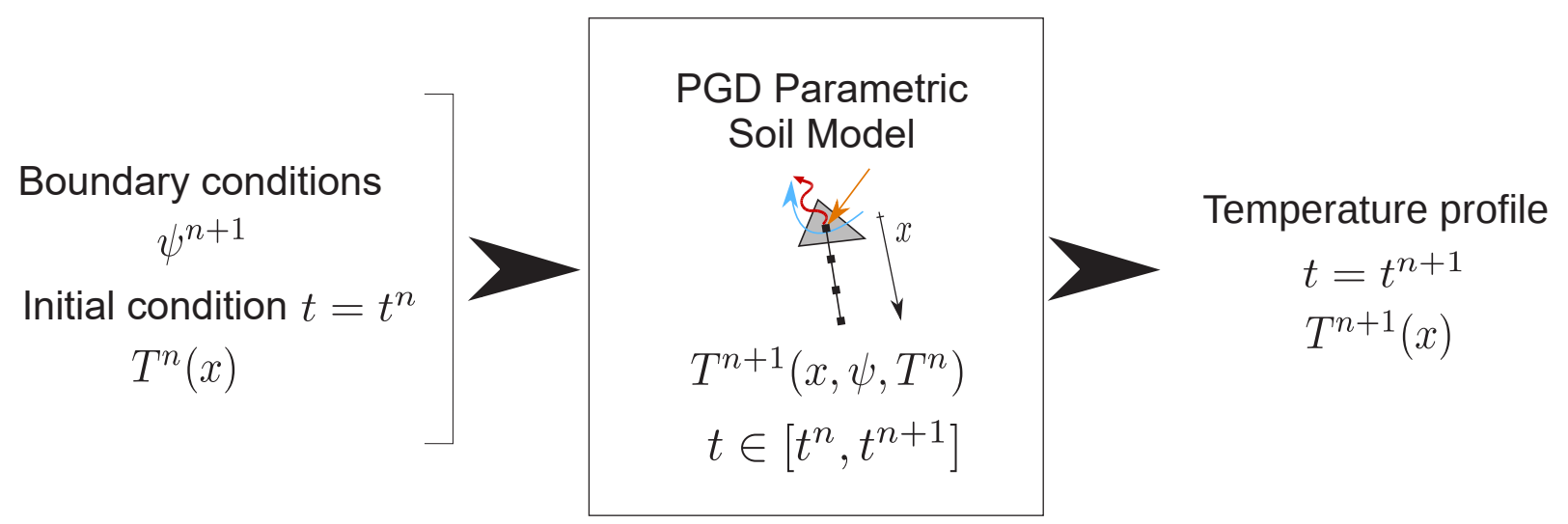

Figure 2: PGD Parametric soil model with the initial condition as a parameter

To achieve a universal solution to this problem, the model boundary conditions need to be defined as parameters. This means that the PGD parametric reduced-order model must be calculated for any boundary conditions over a predefined interval. Given the temperature profile at time step $t^{n}$ and the boundary conditions at time step $t^{n+1}$ as parameters, the model outputs the temperature profile at time step $t^{n+1}$ (Figure 2). This previous temperature profile can be seen as an initial condition of the problem solved between $t^{n}$ and $t^{n+1}$. The model can be developed as a space (x) - boundary conditions (BC) - initial condition (IC) compartmentalization of the solution.

The implicit semi-discretization in time of Eq. (1) yields:

$$
T^{n+1}=T^{n}+\frac{\Delta t}{c} \frac{\partial}{\partial x}\left(k \frac{\partial T^{n+1}}{\partial x}\right),
$$

with the following boundary conditions at $x=0$ :

$$
-k \frac{\partial T^{n+1}}{\partial x}=\psi^{n+1} .
$$

The challenge therefore is to compute a parametric model for the field $T^{n+1}$ by solving problem (2) in searching for a separate solution that depends on space (x) - boundary conditions (BC) - initial condition (IC) as follows:

$$
T^{n+1}=\sum_{i=1}^{\mathcal{M}} X_{i}(x) G_{i}\left(\psi^{n+1}\right) F_{i}\left(T^{n}\right)
$$

It may speculated why a space-time-BC separation of the solution would not suffice. In our specific case, the $\mathrm{BC}$ are not constant over time. The surface energy balance is not the 
same between time steps $t^{n}$ and $t^{n+1}$. With a parametric space-time-BC, the model would compute the solution at $t^{n+1}$ with the $\mathrm{BC}$ at time $t^{n+1}$, from the temperature profile at $t^{n}$. However, the previous temperature profile $\left(\right.$ at $\left.t^{n}\right)$ is calculated with different boundary conditions (at $t^{n+1}$ and not at $t^{n}$ ). The parametric formulation of the problem thus needs to take into account the previous temperature profile as a parameter.

\subsubsection{Use of the POD to parameterize the initial condition}

As presented above, the parametric formulation of the soil problem requires taking the previous temperature profile into account as a parameter. Once discretized in space however, the temperature profile of the initial condition is no longer of infinite dimension: the description of the previous temperature profile provides one piece of information per node, which implies inputting as many parameters in the PGD parametric model as the number of nodes in the grid, plus the boundary conditions and spatial coordinates. To avoid this tremendous number of parameters, the initial condition needs to be parameterized. Gonzalez et al. (2014, [20]) and Cueto et al. (2016, [18]) proposed implementing the Proper Orthogonal Decomposition (POD) method to parametrize the initial condition with a minimum number of parameters. The POD method extracts the relevant information from a set of snapshots by means of its projection into a smaller subspace. As a result, from a set of random data, the POD builds a deterministic representation of a dataset. This representation is built from the basis $\Phi$. The ultimate goal is to retain a detailed representation of the dataset with a minimum or optimal number of modes in $\Phi$. For these properties, the POD method parameterizes the initial temperature profile. More details on the procedure for building the basis $\Phi$ can be found in Appendix B.

This method consists of seeking a set of basis functions $\Phi$ that approximate the temperature profile $T(x, t)$ from the eigenvalues and eigenmodes. The basis $\Phi$ is then used to parameterize the initial condition. For this purpose, the initial system of equations is projected into the reduced-order basis by simply performing the following change of variable:

$$
T^{n}(x) \simeq \tilde{T}^{n}(x)=\sum_{j=1}^{\mathcal{N}} \Phi_{j}(x) \zeta_{j}
$$




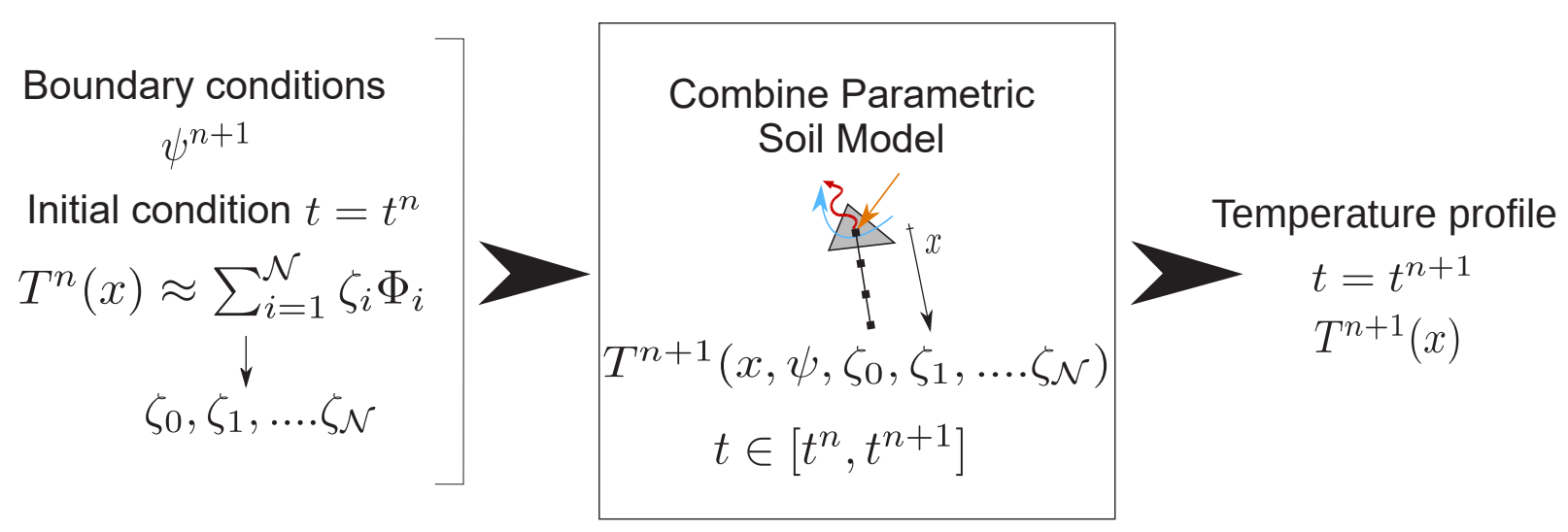

Figure 3: PGD Parametric soil model with the use of POD to configure the initial condition

In this manner, the temperature profile can be optimally parameterized with a minimum number of modes. The previous parametric problem is thus modified as follows:

$$
T^{n+1}=\sum_{i=1}^{\mathcal{M}} X_{i}(x) G_{i}\left(\psi^{n+1}\right) F_{i}^{1}\left(\zeta_{1}\right) F_{i}^{2}\left(\zeta_{2}\right) \ldots F_{i}^{\mathcal{N}}\left(\zeta_{\mathcal{N}}\right)
$$

in which $\zeta_{j}$ are the new parameters of the PGD model, as presented in Figure 3.

\subsubsection{Building the PGD parametric solution}

The PGD method approximates the solution to a problem as a finite sum of separable functions. Such functions are determined by means of an iterative procedure. The PGD can be described according to three main steps, i.e.:

1. initialization of the function basis,

2. enrichment of the basis functions through an iterative process,

3. solution convergence test.

The PGD parametric method will be described hereafter. For further details on the method and its developments, the interested reader may refer to [18, 19]. The model is developed as a space - BC - IC separation of the solution. The boundary condition is defined as a universal function $\psi$ and could be:

- the DiRichlet boundary condition in the ground, 
- the Robin boundary condition at the surface.

We are now searching for the temperature profile:

$$
T^{n+1}\left(x, T^{n}, \psi\right)=\sum_{i=1}^{\mathcal{M}} X_{i}(x) \otimes F_{i}\left(T^{n}\right) \otimes G_{i}(\psi)
$$

where $\left.\left.T \in \Omega_{x} \times\right] 0, \Delta t\right] \times \Omega_{T^{n}} \times \Omega_{\psi}$ with $\Omega_{T^{n}}=\left[T^{n-}, T^{n+}\right]$ and $\Omega_{\psi}=\left[\psi^{-}, \psi^{+}\right]$. The PGD approximation is the sum of $\mathcal{M}$ functional products $\otimes$ involving each function, hereafter denoted by a point in the equations. First, the initial condition $T^{n}$ needs to be introduced explicitly into the weak form of the problem (Eq. (4)), yielding Eq. (5), for an implicit scheme with the test function $u^{*}$ (Eq. (6)). This test function formulation (i.e. Galerkin formulation) is most frequently used in the literature:

$$
\int_{\Omega_{x} \times \Omega_{T} n \times \Omega_{\psi}} u^{*} \cdot\left(c \frac{\partial T}{\partial t}-\frac{\partial}{\partial x}\left(k \frac{\partial T}{\partial x}\right)\right) \mathrm{d} x \cdot \mathrm{d} T^{n} \cdot \mathrm{d} \psi=0
$$

$$
\begin{gathered}
\int_{\Omega_{x} \times \Omega_{T} \times \Omega_{\psi}} u^{*} \cdot\left(c \frac{T^{n+1}-T^{n}}{\Delta t}-\frac{\partial}{\partial x}\left(k \frac{\partial T^{n+1}}{\partial x}\right)\right) \mathrm{d} x \cdot \mathrm{d} T^{n} \cdot \mathrm{d} \psi=0 \\
u^{*}\left(x, T^{n}, \psi\right)=X^{*}(x) \cdot F\left(T^{n}\right) \cdot G(\psi)+X(x) \cdot F^{*}\left(T^{n}\right) \cdot G(\psi)+X(x) \cdot F\left(T^{n}\right) \cdot G^{*}(\psi)
\end{gathered}
$$

The separated representation is built with an iterative procedure that features two nested loops: the alternating direction strategy, and the enrichment process. At enrichment step $m$, the first $m-1$ terms have been computed. The new functions $X_{m}, F_{m}$ and $G_{m}$ must now be calculated according to:

$$
T_{m}^{n+1}\left(x, T^{n}, \psi\right)=\sum_{i=1}^{m-1} X_{i}(x) \cdot F_{i}\left(T^{n}\right) \cdot G_{i}(\psi)+X_{m}(x) \cdot F_{m}\left(T^{n}\right) \cdot G_{m}(\psi)
$$

A nonlinear problem must now be solved where the unknowns are the functions $X_{m}, F_{m}$ and $G_{m}$. In this aim, an alternating direction, also called a fixed-point algorithm, will be used. Each function is randomly initialized and then solved by iteration. At enrichment step $m$ and at iteration $p$ of the fixed-point algorithm, we obtain the following temperature profile approximation:

$$
T_{m}^{n+1, p}\left(x, T^{n}, \psi\right)=T_{m-1}^{n+1}\left(x, T^{n}, \psi\right)+X_{m}^{p}(x) \cdot F_{m}^{p}\left(T^{n}\right) \cdot G_{m}^{p}(\psi)
$$


As mentioned above, the PGD algorithm is composed of two enrichment loops. Each step is summarized in Algorithm 1 and detailed thereafter.

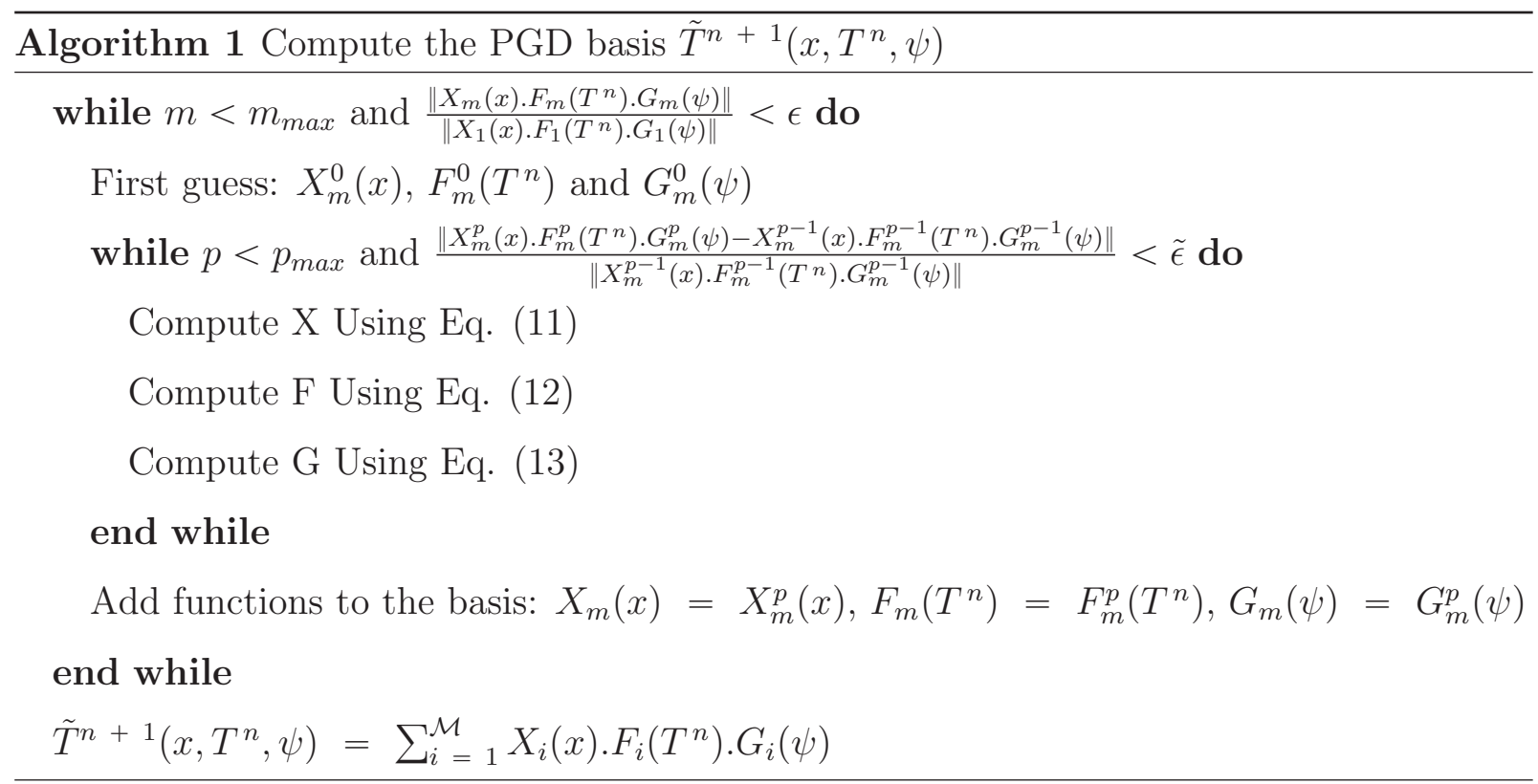

First, the fixed-point algorithm is randomly initialized, then each term is calculated one after the other:

- $X_{m}^{p}(x)$ with $F_{m}^{p-1}\left(T^{n}\right)$ and $G_{m}^{p-1}(\psi)$ assumed to be known;

- $F_{m}^{p}\left(T^{n}\right)$ with $X_{m}^{p}(x)$ and $G_{m}^{p-1}(\psi)$ assumed to be known;

- $G_{m}^{p}(\psi)$ with $X_{m}^{p}(x)$ and $F_{m}^{p}\left(T^{n}\right)$ assumed to be known.

The alternating direction process stops once a fixed point has been reached. The criterion used to make this determination is defined in Eq. (9) with $\tilde{\epsilon}$ being a criterion defined by the user [19].

$$
\frac{\left\|X_{m}^{p}(x) \cdot F_{m}^{p}\left(T^{n}\right) \cdot G_{m}^{p}(\psi)-X_{m}^{p-1}(x) \cdot F_{m}^{p-1}\left(T^{n}\right) \cdot G_{m}^{p-1}(\psi)\right\|}{\left\|X_{m}^{p-1}(x) \cdot F_{m}^{p-1}\left(T^{n}\right) \cdot G_{m}^{p-1}(\psi)\right\|}<\tilde{\epsilon}
$$

Upon completion of the fixed-point algorithm, the functions are added to the basis: $X_{m}(x)=X_{m}^{p}(x), F_{m}\left(T^{n}\right)=F_{m}^{p}\left(T^{n}\right), G_{m}(\psi)=G_{m}^{p}(\psi)$. The enrichment process of the PGD basis stops when the following criterion $\epsilon$, defined by the user, has been reached (Eq. (10)) [19]. 


$$
\frac{\left\|X_{m}(x) \cdot F_{m}\left(T^{n}\right) \cdot G_{m}(\psi)\right\|}{\left\|X_{1}(x) \cdot F_{1}\left(T^{n}\right) \cdot G_{1}(\psi)\right\|}<\epsilon
$$

\section{Alternation direction strategy}

The first step of the fixed-point algorithm has now been described; it consists of computing $X_{m}^{p}(x)$. Since the methodology is the same for computing $F_{m}^{p}\left(T^{n}\right)$ and $G_{m}^{p}(\psi)$, the two following steps will be developed in the Appendix.

Computation of $X_{m}^{p}(x)$ from $F_{m}^{p-1}\left(T^{n}\right)$ and $G_{m}^{p-1}(\psi)$

Eq. (7) is introduced into Eq. (5), along with the following test function:

$$
u^{*}\left(x, T^{n}, \psi\right)=X_{m}^{*}(x) \cdot F_{m}^{p-1}\left(T^{n}\right) \cdot G_{m}^{p-1}(\psi)=X^{*} \cdot F \cdot G
$$

We then obtain the following equation:

$$
\begin{array}{r}
\int_{\Omega_{x} \times \Omega_{T} n \times \Omega_{\psi}} X^{*} \cdot F \cdot G\left(c \frac{X \cdot F \cdot G}{\Delta t}-\frac{\partial}{\partial x}\left(k \frac{\partial X}{\partial x}\right) \cdot F \cdot G\right) \mathrm{d} x \cdot \mathrm{d} T^{n} \cdot \mathrm{d} \psi \\
-\int_{\Omega_{x} \times \Omega_{T} n \times \Omega_{\psi}} X^{*} \cdot F \cdot G \cdot c \frac{T^{n}}{\Delta t} \mathrm{~d} x \cdot \mathrm{d} T^{n} \cdot \mathrm{d} \psi \\
=-\int_{\Omega_{x} \times \Omega_{T} n \times \Omega_{\psi}} X^{*} \cdot F \cdot G \sum_{i=1}^{m-1}\left(c \frac{X_{i} \cdot F_{i} \cdot G_{i}}{\Delta t}-\frac{\partial}{\partial x}\left(k \frac{\partial X_{i}}{\partial x}\right) \cdot F_{i} \cdot G_{i}\right) \mathrm{d} x \cdot \mathrm{d} T^{n} \cdot \mathrm{d} \psi
\end{array}
$$

Since all the functions depending on the parametric coordinate $\psi$ and $T^{n}$ are known, they can be integrated over their domain: $\Omega_{T^{n}}$ and $\Omega_{\psi}$.

$$
\left\{\begin{array}{rrrr}
f_{1} & = & & \int_{\Omega_{T} n}(F) \mathrm{d} T^{n} \\
f_{2} & = & \int_{\Omega_{T} n}(F)^{2} \mathrm{~d} T^{n} \\
g_{1} & = & \int_{\Omega_{\psi}}(G) \mathrm{d} \psi \\
g_{2} & = & & \int_{\Omega_{\psi}}(G)^{2} \mathrm{~d} \psi \\
f_{i} & = & \int_{\Omega_{T^{n}}}\left(F \cdot F_{i}\right) \mathrm{d} T^{n} \\
g_{i} & = & \int_{\Omega_{\psi}}\left(G \cdot G_{i}\right) \mathrm{d} \psi
\end{array}\right.
$$

We derive the following simplified equation (11), which can be solved by any discretization technique. The finite difference method has been used herein, with a center second-order derivative scheme. 
$c \frac{X}{\Delta t} \cdot f_{2} \cdot g_{2}-\frac{\partial}{\partial x}\left(k \frac{\partial X}{\partial x}\right) \cdot f_{2} \cdot g_{2}=-\sum_{i=1}^{m-1}\left(c \frac{X_{i}}{\Delta t} \cdot f_{i} \cdot g_{i}-\frac{\partial}{\partial x}\left(k \frac{\partial X_{i}}{\partial x}\right) \cdot f_{i} \cdot g_{i}\right)+f_{1} \cdot g_{1} \cdot c \frac{T^{n}}{\Delta t}$

With this same method, $F_{m}^{p}\left(T^{n}\right)$ can be computed from $X_{m}^{p}(x)$ and $G_{m}^{p-1}(\psi)$, producing the following algebraic equation (12), whose direct solution yields the function $F$. Details on this development can be found in Appendix C.1.

$$
F \cdot\left(\frac{c}{\Delta t} x_{2} g_{2}-x_{3} g_{2}\right)=-\sum_{i=1}^{m-1}\left(c \frac{F_{i}}{\Delta t} x_{i, 1} g_{i}-F_{i} x_{i, 2} g_{i}\right)+x_{1} g_{1} c \frac{T^{n}}{\Delta t}
$$

Moreover, $X_{m}^{p}(x)$ and $F_{m}^{p}\left(T^{n}\right)$ enable computing $G_{m}^{p}(\psi)$. Equation (13) presents the final algebraic equation; its direct solution yields the function $G$. Details on this development can be found in Appendix C.2.

$$
G\left(\frac{c}{\Delta t} x_{2} f_{2}-x_{3} f_{2}\right)=-\sum_{i=1}^{m-1}\left(c \frac{G_{i}}{\Delta t} \cdot x_{i, 1} \cdot f_{i}-G_{i} x_{i, 2} f_{i}\right)+x_{1} f_{1} c \frac{T^{n}}{\Delta t}
$$

\subsection{Implementation methodology}

The combined parametric soil model developed herein makes use of both POD and PGD methods and features an offline/online strategy. All steps are detailed in Figure 4. The POD method serves to configure the previous soil temperature profile, as presented in Figure 3. In order to perform this configuration, we first need a POD basis, which is built from a set of snapshots calculated by the LOM (via the finite difference method). This basis is calculated once offline. For each of the equations (11), (12), and (13), the previous temperature profile is projected into a POD basis by executing the following change of variable:

$$
T^{n} \approx \sum_{i=1}^{\mathcal{N}} \zeta_{i} \Phi_{i}=\Phi \zeta
$$

A minimum number of modes in the POD basis, $\mathcal{N}$, is defined to achieve the desired accuracy. Note that the number of modes has a direct influence on the number of parameters used in the PGD model. The parametric model can then be solved with the PGD Algorithm 1 for: 


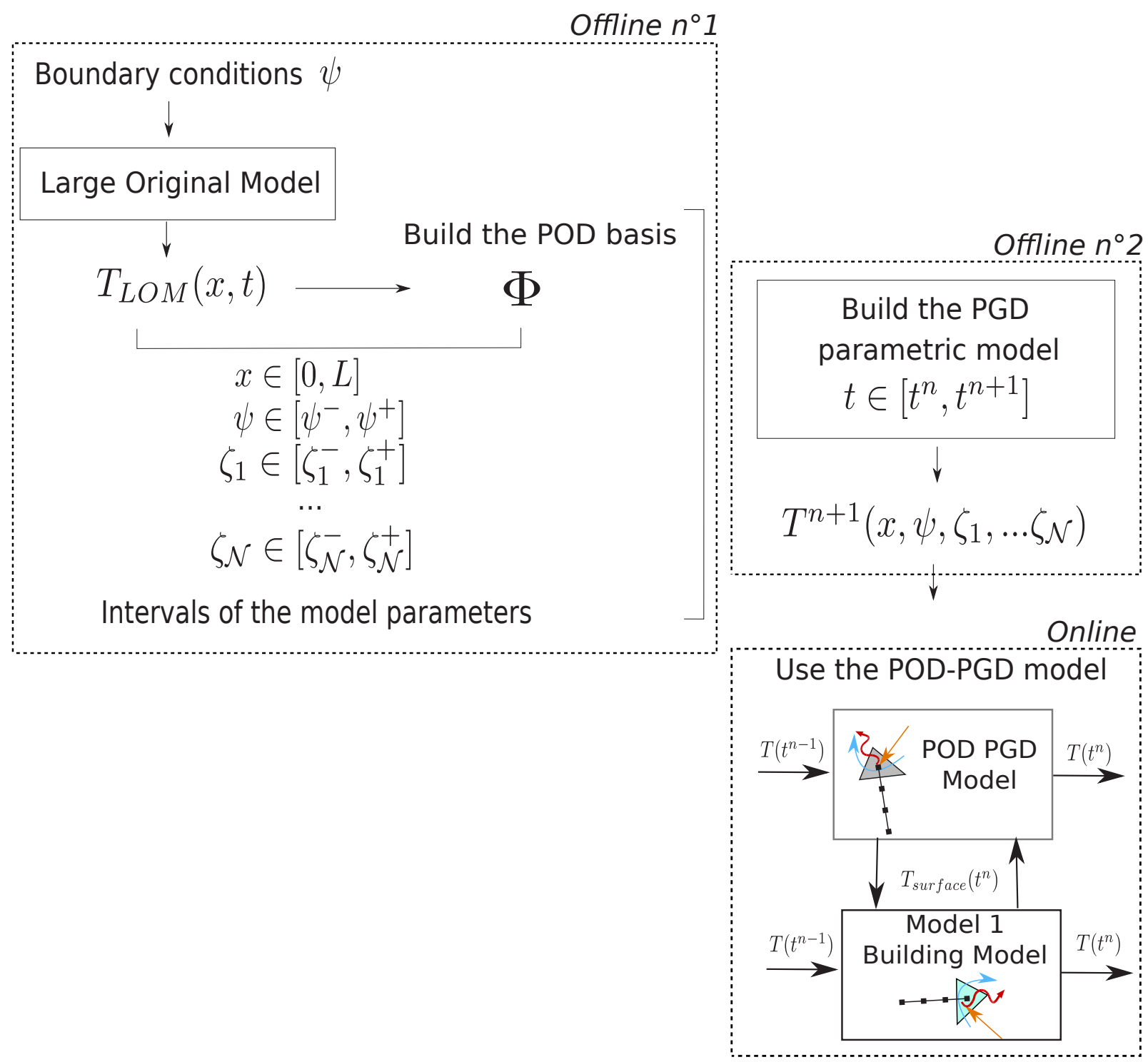

Figure 4: Implementation methodology of the model 
- a spatial mesh,

- a time interval of $\left(T^{n}, t^{n+1}\right)$,

- any values of the boundary condition $\psi$ defined within a discretized interval $\left[\psi^{-}, \psi^{+}\right]$,

- each mode of the POD basis: i.e. $\zeta_{1} \in\left[\zeta_{1}^{-}, \zeta_{1}^{+}\right], \ldots \zeta_{\mathcal{N}} \in\left[\zeta_{\mathcal{N}}^{-}, \zeta_{\mathcal{N}}^{+}\right]$.

Once the PGD parametric model has been built, it can be applied for any value within the previously defined intervals. Figure 3 summarizes the utility of the POD+PGD parametric model. The initial condition is projected onto the reduced basis $\Phi$ so as to identify the parameters $\zeta_{i}$ at time step $T^{n}$. Afterwards, the PGD modes are computed for the defined parameters $x, \zeta_{i}$ and $\psi$.

\subsection{Assessment methodology}

The global methodology used to assess this model is presented herein. The combined parametric model is applied to several study cases in order to evaluate the numerical method and the ability of the model to properly reproduce physical phenomena with or without influence of the urban environment. The results of the developed POD+PGD parametric soil model $T_{\text {num }}(x, t)$ are then compared to the results of a reference solution $T_{r e f}(x, t)$ that could be analytical, measurements or the LOM results. For each case study, the indicator chosen is the $\ell_{2}$ norm (or Root Mean Square Error) noted $\varepsilon_{2}$; it is computed as a spatial or time function by the following discrete $\ell_{2}$ formulation, where $N_{x}$ and $N_{t}$ are the number of elements over each axis.

$$
\begin{aligned}
& \varepsilon_{2}(t)=\sqrt{\frac{1}{N_{x}} \sum_{0}^{N_{x}}\left[T_{\text {num }}(x, t)-T_{\text {ref }}(x, t)\right]^{2}} \\
& \varepsilon_{2}(x)=\sqrt{\frac{1}{N_{t}} \sum_{0}^{N_{t}}\left[T_{\text {num }}(x, t)-T_{\text {ref }}(x, t)\right]^{2}}
\end{aligned}
$$

The global error is given by the maximum of the previous function $\varepsilon_{2}(t)$ and $\varepsilon_{2}(x)$ as described hereafter:

$$
\varepsilon_{\infty}=\max _{t}\left(\varepsilon_{2}(x)\right)
$$




\section{Results}

\subsection{Evaluation of the numerical method with an analytical solution}

In this part, for validation purposes, the POD+PGD ROM will be applied to a case containing an analytical solution. The respective performances of this new model, the finite difference model and the POD model on its own will be compared. The previously defined problem will be solved for a uniform slab with a thermal diffusivity of $6.10^{-7} \mathrm{~m}^{2} . \mathrm{s}^{-1}$ and conductivity of $1.5 \mathrm{~W} \cdot \mathrm{m}^{-1} \cdot \mathrm{K}^{-1}$. The slab body is initially at a constant temperature, $T(x, 0)=0$. The temperature at $x=L$ is set at $T(L, t)=0$. At the surface $(x=0)$, only a convective heat flux is considered, with: a cosine-periodic air temperature whose amplitude is $10^{\circ} \mathrm{C}$, a period of 24 hours, and a convective heat transfer coefficient of $10 \mathrm{~W} \cdot \mathrm{m}^{-2} \cdot \mathrm{K}^{-1}$. No source term has been taken into account. The spatial domain $\Omega_{x}=[0,1]$ is discretized with a uniform mesh $d x=0.01 \mathrm{~m}$. The total simulation time lasts two days, with a time step of 15 minutes. Only the last day is studied, while the first day is considered as the period necessary to initialize the model. The problem is first solved by running the finite difference method. Then, the POD model is built on the previous results with four nodes $(\mathcal{N}=4)$. The PGD parametric model is built with both the initial condition and air temperature as parameters $\left(\psi=T_{\text {air }}\right)$ :

$$
T^{n+1}\left(x, \zeta_{1}, \zeta_{2}, \zeta_{3}, \zeta_{4}, T_{a i r}\right)=\sum_{i=1}^{\mathcal{M}} X_{i}(x) \cdot C_{i}\left(\zeta_{1}\right) \cdot D_{i}\left(\zeta_{2}\right) \cdot E_{i}\left(\zeta_{3}\right) \cdot F_{i}\left(\zeta_{4}\right) \cdot G_{i}\left(T_{\text {air }}\right) .
$$

Each computed solution is compared to an analytical solution from the EXACT toolbox [21] described in the Appendix A. Figure 5 shows the evolution of temperature calculated by the analytical solution as well as by the three developed models. The temperature signal of the Finite Difference model and the reduced-order model (POD+PGD parametric) overlap. As revealed in these figures, the ROM solutions with 4 modes and the Finite Difference model provide similar results with $\epsilon_{2}=0.03^{\circ} \mathrm{C}$ at the surface and $0.01^{\circ} \mathrm{C}$ at a depth of $0.5 \mathrm{~m}$. Figure 6 indicates the $\ell_{2}$ error evolution for the various models compared to the analytical solution. The quantity $\varepsilon_{\infty}$ equals $0.045^{\circ} \mathrm{C}$ for the combined parametric model. Since the POD basis is used under similar conditions to those for the learning process, the results of the combined method provide a close fit. The third set of errors given in Figure 6 estimates the error due 


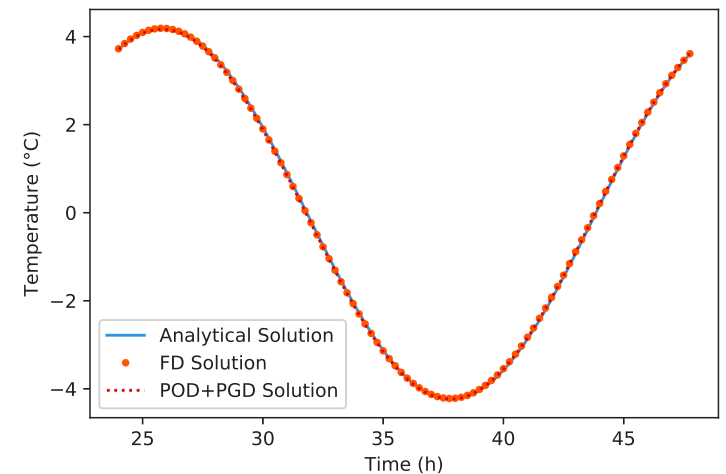

(a) Surface temperature evolution

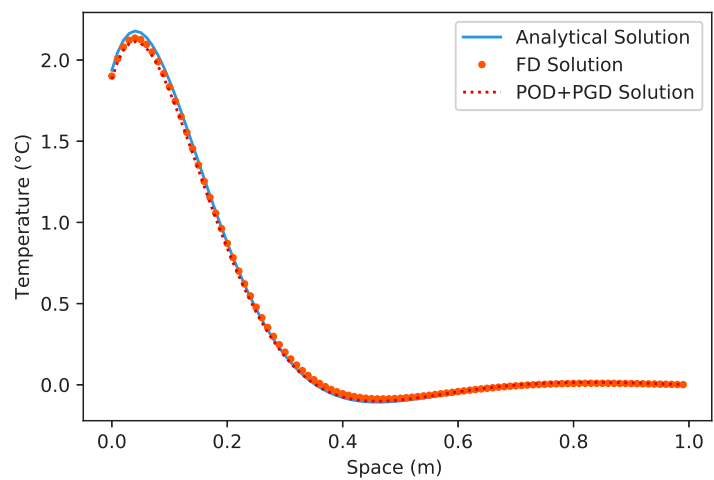

(b) Temperature profile $(25 \mathrm{~h})$

Figure 5: Temperature profile calculated by the analytical solution as well as by the FD and combined parametric models

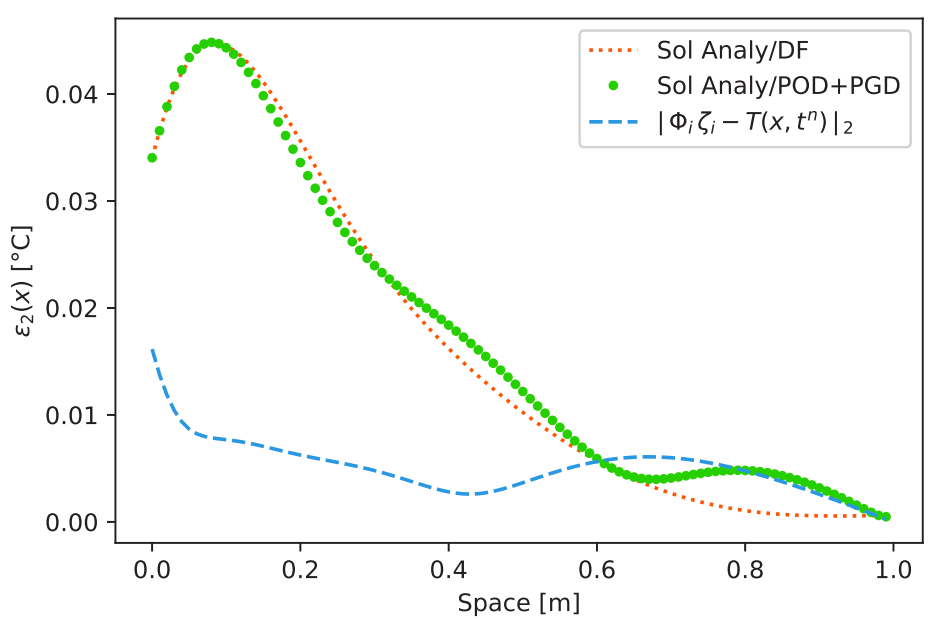

Figure 6: Evolution of the $\ell 2$ error. First set: calculated between the Analytical Solution and the Finite Difference model. Second set: calculated between the POD+PGD parametric model and the Analytical Solution. Third set: estimate drawn from the error due to the POD parameterization of the initial condition 


\subsection{Application to a case study independent of the influence of the urban environment}

The combined parametric model will be applied in this section to an asphalt parking lot. This layout was chosen to avoid interactions with other surfaces due to solar and longwave radiative exchanges with surrounding vertical surfaces (building facades). Under these conditions, the POD+PGD parametric model can be evaluated before its coupling with the micro-climatic model. Results of the combined parametric model will be compared to measurements conducted on this lot. The measurement campaign will be presented first, then the model will be applied and assessed.

\subsubsection{Presentation of the measurement campaign}

Data from the ROSURE/HydroVille project [22] are used herein. The experimental site is located near Nantes (France) and consists of an asphalt parking lot measuring $2500 \mathrm{~m}^{2}$. This campaign has entailed surface and air temperatures as well as on heat flux measurements during a warm summer period (June 2004). During the experiment, the parking lot was watered to simulate artificial rain events. For the observations available during this campaign, this paper focuses on all variables measured at the middle of the lot:

- surface and ground temperatures: vertical profiles at depths of $0,1,2,3,4,5,6,10$, $15,24,34,50$ and $75 \mathrm{~cm}$;

- wind speed;

- radiation components.

The humidity and air temperatures were measured outside the lot. The data were collected with a 1-min time step. The final data were averaged over 15-min intervals. More information on this campaign can be found in [22].

\subsubsection{Combined parametric model set-up}

The simulation is run for the entire period from June $5^{\text {th }}$ at midnight to June $14^{\text {th }}$ midnight, with a 15 - minutes time step. A centimetric grid is used. The temperature profile is initialized from ground temperatures measured on June $4^{\text {th }}$ at 11:45 pm. The boundary depth condition, which corresponds to the ground temperature at a depth of $75 \mathrm{~cm}$ has 


\begin{tabular}{lll} 
Heat fluxes & Input data used to calculate each flux & Calculation Method \\
\hline$q_{c}$ & Measured wind speed & Correlation method \\
& Measured surface temperature & \\
& Measured air temperature & Measurements \\
\hline$q_{n e t}$ & Measured net radiative flux & Mass-transfer method \\
\hline$q_{\ell}$ & Water height & \\
& Water-holding capacity of the surface & \\
& Measured air relative humidity & Water layer energy balance \\
& Measured air temperature & \\
\hline$q_{w}$ & Measured water temperature & \\
& Total measured total sprinkled water & \\
\hline
\end{tabular}

Table 1: Detail of each input data required to calculate the upper boundary condition

been set in accordance with the experimental data. At the surface, a NEumann boundary condition is considered from the surface energy balance detailed below:

$$
-k \frac{\partial T}{\partial x}=q_{n e t}-q_{c}-q_{\ell}-q_{w}
$$

The convective heat flux $q_{c}$ is calculated from the measured air and surface temperature plus the wind speed using a correlation method with a characteristic length of $1 \mathrm{~m}$. More details on this method can be found in [10]. For the radiative heat fluxes $q_{\text {net }}$, the net heat flux measurement is used. During a watering event, two heat fluxes are to be added to the previous ones: a runoff convective heat flux $q_{w}$, and a latent heat flux $q_{\ell}$. The computational details of these two fluxes is described in [23]. The runoff convective heat flux is calculated from the amount of water sprinkled during each watering event. Data acquired on June $7^{\text {th }}$, and $8^{\text {th }}$ were used to calibrate the surface water holding capacity. The latent heat flux is calculated from measured air characteristics (temperature, relative humidity). All of the input data categories are listed in the Table 1.

The soil is composed of three different materials: $5 \mathrm{~cm}$ of asphalt, $45 \mathrm{~cm}$ of ballast, and an altered natural mica-schist soil underneath. The soil composition and thermal properties were not measured during the campaign; instead, they were calibrated according to the observed soil thermal profile, thus reducing the difference between measured and simulated 
surface temperature on the centimetric grid. The data measured on June $6^{\text {th }}$ were used for calibration purposes. From the measured temperature gradient, changes in soil thermal properties in the first layer were identified $(0-1 \mathrm{~cm}, 1-5 \mathrm{~cm})$. The calibrated soil properties are summarized in Table 2.

\begin{tabular}{|c|c|c|c|}
\hline $\begin{array}{l}\text { Layer depth } \\
\mathrm{m}\end{array}$ & $\begin{array}{l}\text { Material } \\
\text { Characteristics }\end{array}$ & $\begin{array}{l}\text { Thermal conductivity } \\
W \cdot m^{-1} \cdot K^{-1}\end{array}$ & $\begin{array}{l}\text { Volumetric heat capacity } \\
10^{6} J \cdot m^{-3} \cdot K^{-1}\end{array}$ \\
\hline $0.00-0.01$ & Asphalt Concrete & 2.5 & 2.3 \\
\hline $0.01-0.05$ & Asphalt Concrete & 2.5 & 2.1 \\
\hline $0.05-0.50$ & Old Filled Ballast & 1.8 & 2.3 \\
\hline $0.50-0.75$ & Natural Soil & 1.3 & 2.1 \\
\hline
\end{tabular}

Table 2: Calibrated soil characteristics

As presented in Section 2.3, the problem is first solved using the finite difference method. Next, the POD model is built on the previous results with four nodes $(\mathcal{N}=4)$. The PGD parametric model is built with the initial condition, the surface heat flux $q_{a}$ and the ground temperature $T_{\infty}$ all as parameters, whereas the PGD parametric model comprises 16 modes $(\mathcal{M}=16)$. The combined parametric model is thus as follows:

$$
\tilde{T}^{n+1}\left(x, \zeta_{1}, \zeta_{2}, \zeta_{3}, \zeta_{4}, q_{a}, T_{\infty}\right)=\sum_{i=1}^{\mathcal{M}} X_{i}(x) \cdot C_{i}\left(\zeta_{1}\right) \cdot D_{i}\left(\zeta_{2}\right) \cdot E_{i}\left(\zeta_{3}\right) \cdot F_{i}\left(\zeta_{4}\right) \cdot G_{i}\left(q_{a}\right) \cdot H_{i}\left(T_{\infty}\right)
$$

\subsubsection{Combined parametric model assessment}

The ROM model is then evaluated by comparing the time series of the measured and calculated temperatures at the surface and at several depths within the soil. Since the proposed model has combined an a priori method (PGD) with an a posteriori one (POD), a learning process is required. This process consists of building the POD basis just once and offline. The combined parametric model can then be used online, under different heat boundary conditions. To obtain an efficient combined parametric model, this learning period should be as short as possible. At first, in order to evaluate model performance under actual conditions, the learning and simulation periods will be the same. This initial study is aimed at assessing model behavior regardless of the influence from the learning period. 


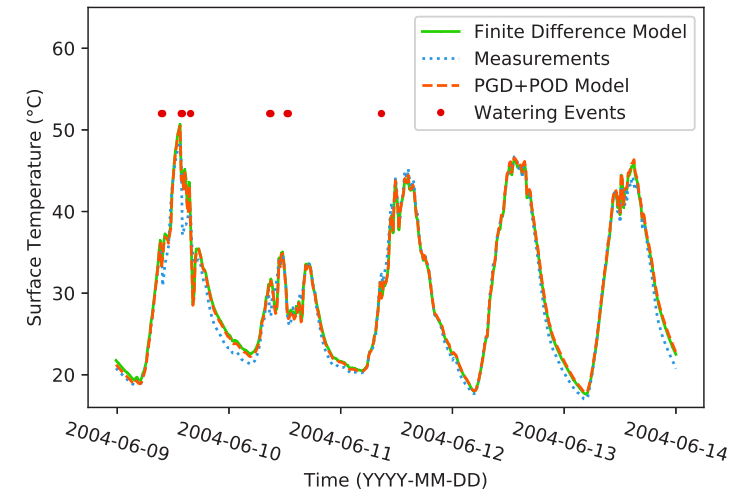

(a) Comparison of the surface temperatures from June $9^{\text {th }}$ to $13^{\text {th }}$

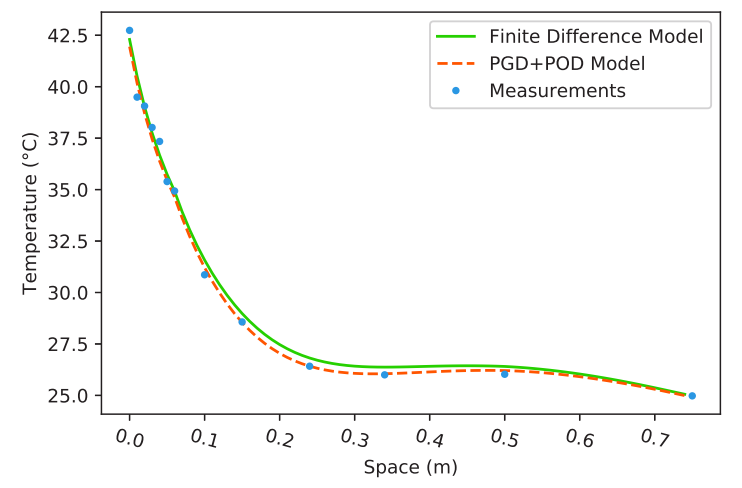

(b) Comparison of the temperature profiles from June $9^{\text {th }}$ at 11:45 am (watering event at 09:30 am)

Figure 7: Comparison of simulated and measured temperatures

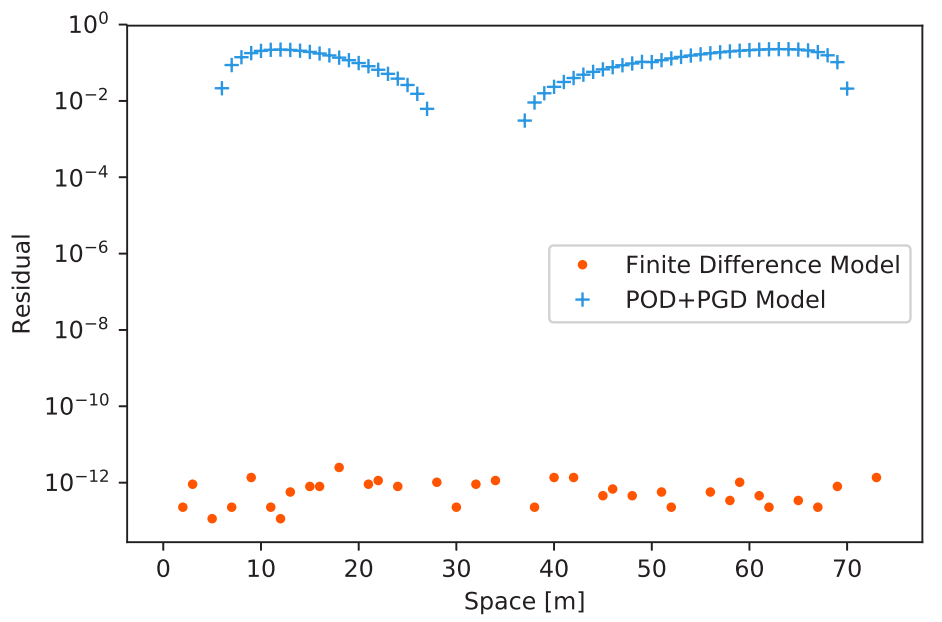

Figure 8: Comparison of the residual of the heat equation calculated for both methods at the last time step 
In this instance, the ROM model is evaluated over an entire learning period. For both models studied, the temporal variations in the surface temperature are well reproduced compared to the measurement results (Figure 7). The combined parametric model signal is closer to the measurement signal than that produced by the finite difference model. In considering this observation, the combined parametric model might seem more accurate than the finite difference model. If we were to compare the numerical residuals of both methods however, the final residual given by the finite difference method would be smaller than that output by the ROM (Fig. 8). According to these results, the PGD solution cannot then be more accurate than the finite difference solution. Due to the calibration of material properties, the finite difference model itself overestimates the surface temperature, whereas the reduced order model underestimates it.

During watering events, the same shape is obtained yet with a time lag. This error is independent of the reduced order model behavior itself but depends on the surface energy balance. As described in [23], the surface energy balance during a watering event takes into account additional heat fluxes: a latent heat flux and a runoff convective heat flux. The dynamics of both these heat fluxes are complicated to reproduce during a watering event, which explains the observed time lag. This discrepancy is due to an approximation of the physical phenomena in the model and not in the numerical solution.

To determine overall ROM performance, four sets of errors are calculated. The results are presented in Figure 9. The first set evaluates the overall accuracy of the combined parametric model. The quantity is calculated between the POD+PGD and measured temperatures. For the sake of comparison, a second set has been calculated between the finite difference model and the measurements. These first two sets provide the total error due to the chosen numerical scheme, the numerical method and the physical model. To evaluate the error due solely to the numerical scheme and method, a third error set evaluates the loss in accuracy due to the reduction in model order; $\epsilon_{2}(x)$ is calculated between the POD+PGD parametric model and the finite difference model. The last set estimates the error due to POD parameterization of the initial condition. These $\ell_{2}$ errors have all been calculated from June $8^{\text {th }}$ at 11:45 pm to June $13^{\text {th }}$ at the same time, outside the calibration period. 


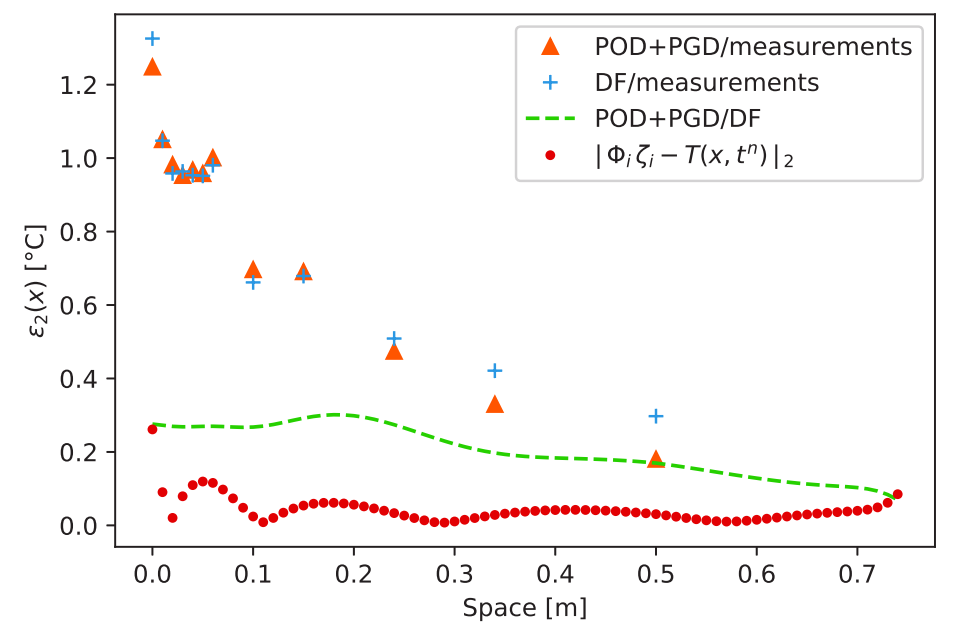

Figure 9: Evolution of the $\ell 2$ error. First set: calculated between the POD+PGD parametric model and the measurements. Second set: calculated between the finite difference model and the measurements. Third set: calculated between the parametric model and the finite difference model. Fourth set: estimated error due to the POD parameterization of the initial condition.

This loss of accuracy due to the model order reduction remains less than $0.30^{\circ} \mathrm{C}$. The error is greater within the first few centimeters, close to the surface boundary condition, and then decreases with depth. The combined parametric model accuracy depends directly on the POD basis accuracy (used to describe the previous temperature profile). The final set in Figure 9 shows that a portion of the error is due to the initial condition parameterization; this error prevails close to the boundary condition, where variations are steeper.

The $\epsilon_{2}(x)$ error between the POD+PGD parametric model and the measurements equals $1.24^{\circ} \mathrm{C}$ at the surface, while it amounts to $1.32^{\circ} \mathrm{C}$ between the finite difference model and the measurements. The combined parametric model might appear to be more accurate, but as explained before the reduced-order model cannot be more accurate since the numerical residual of the POD+PGD method exceeds the finite difference residual. The model order reduction has consequences on the reproduction of daily peaks, specifically it underestimates the temperature signal amplitude. This same phenomenon could be observed with the finite difference model and a coarser grid.

After evaluating the numerical method with the analytical solution, this case study has evaluated both the numerical method and the physical model. Moreover, under actual con- 
ditions, both models (POD+PGD and FD) are capable of reproducing the dynamics with relatively good accuracy. Hence, the combined parametric model can be applied to more complex situations, such as an urban scene.

\subsubsection{Influence the learning period}

As mentioned above, the accuracy of the combined parametric model depends on the accuracy of the POD basis, which is directly determined by the learning period. In order to study the influence of this parameter on model behavior, various learning periods are compared. Figure 10 provides the evolution of the global error as a function of the number of days used for the learning period. The error $\varepsilon_{2}(x)$ is calculated between the combined parametric model and the measurements (crosses) as well as between the combined parametric model and the finite difference model (points). The first set indicates the evolution of the global model error, which pertains to the numerical scheme, plus the methods errors and physical model errors. The second set indicates the evolution of the error due specifically to the learning period. Both sets are nearly constant over the number of days used to compose the learning period. The number of days selected for the learning process has no influence on the maximum observable error. The assumption could thus be made that using a longer learning period will not improve the maximum model error.

The previous indicator however does not yield information on the ability of the model to reproduce a specific dynamic. As such, we are proposing herein to study the model response to various stresses. The dataset examined contains various boundary conditions with sudden drops in the temperature signal due to watering events. Three learning conditions are assessed: one day with no watering events (June $13^{\text {th }}$ ), one day with two watering events (June $10^{\text {th }}$ ), and the full learning period. June $10^{\text {th }}$ and $13^{\text {th }}$ have been drawn from the calibration period.

Figure 11 displays the $\varepsilon_{2}(t)$ of the different learning periods calculated between the parametric model and the finite difference model over time. For these three periods, several observation can be drawn. Over the entire period, the combined parametric model is more accurate when dry days are considered (i.e. June $5-6^{\text {th }}$ and $12-13^{\text {th }}$ ). Watering events are more difficult to represent accurately. When the learning period is June $13^{\text {th }}$ (see Fig. 11), 


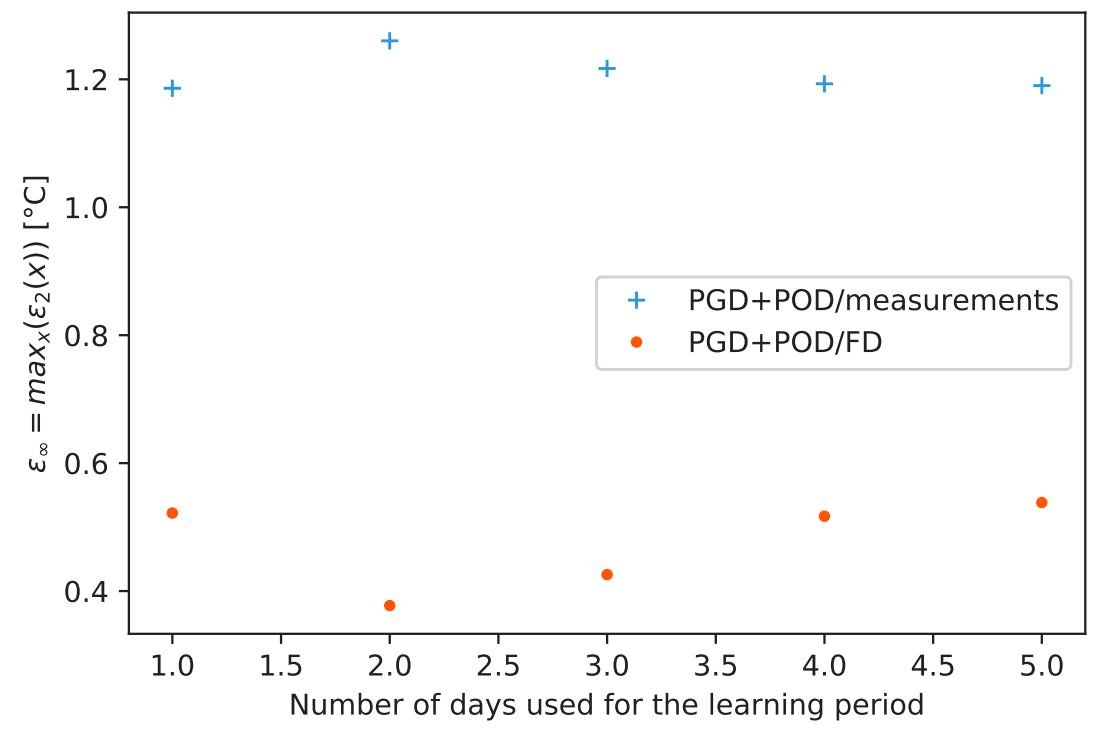

Figure 10: $\varepsilon_{\infty}$ vs. number of days used for the learning period

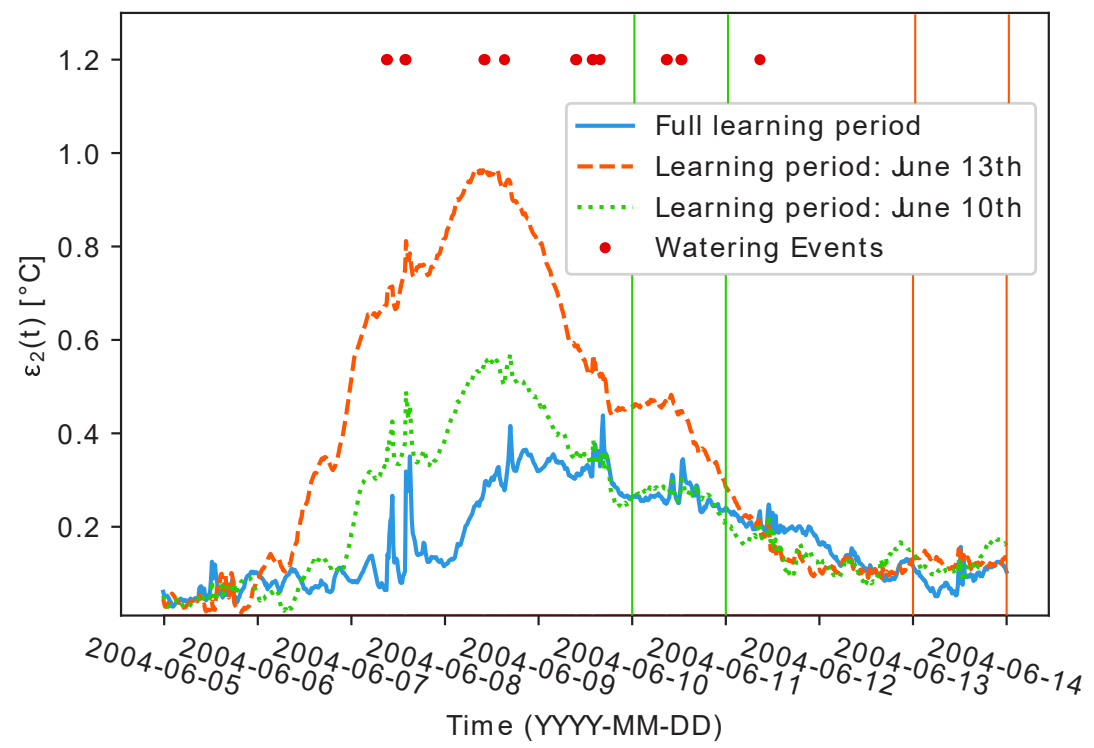

Figure 11: The RMSE calculated between the finite difference model and the POD+PGD parametric model for each temperature profile over time and for different learning periods 
the POD+PGD parametric model is once again accurate under dry conditions (June $5-6^{\text {th }}$ and $12-13^{\text {th }}$ ). The learning period however has been reduced to just a single day, compared to the previous 9 days with the full learning period. Nonetheless, days with watering events still have a higher RMSE value. The combined parametric model is incapable of accurately reproducing this type of boundary condition. Moreover, June $10^{\text {th }}$ is used as the learning period (green rectangle in Fig. 11) and includes watering events. The model therefore is accurate when learning conditions are considered (June $10^{\text {th }}$ ). Yet aside from this period, the model loses accuracy due either to dry days or a watering event.

Since the POD basis is sensitive to the learning period, the learning process and, in our specific case, the period need to be carefully selected. The previous comparison illustrates the difficulty involved in selecting a representative period for all weather conditions extending over a longer time scale (season or year). Since it has been validated under actual boundary conditions, the POD+PGD parametric model can now be applied to an urban scene in order to study the thermal behavior of the model under new constraints.

\subsection{Practical application to an urban environment}

The ROM developed will now be applied to the case of a theoretical urban environment consisting of two canyon streets. For this purpose, the ROM has been coupled with the SOLENE-microclimat simulation tool [6, 7], through use of the ping-pong method [24], which will be discussed in Section 3.3.3. The microclimate tool is used to take into account the surrounding surface in the surface energy budget (i.e. short- and long-wave radiative budget). The objective here is to study the behavior of the ROM model implemented within a complete urban heat balance. In this pursuit, the results of the combined parametric model will be compared to those of the finite difference model under various conditions. The model covers all soil within the urban scene. The study focuses on three zones featuring various radiative stresses. The influence of the area chosen to conduct the learning process will be analyzed, and the computational cost of the various models will be compared.

\subsubsection{Description of the urban scene}

The urban scene is composed of a square and two canyon streets, with an aspect ratio of $H / W=1$ (H: building height; W: street width). The streets are oriented north-south 


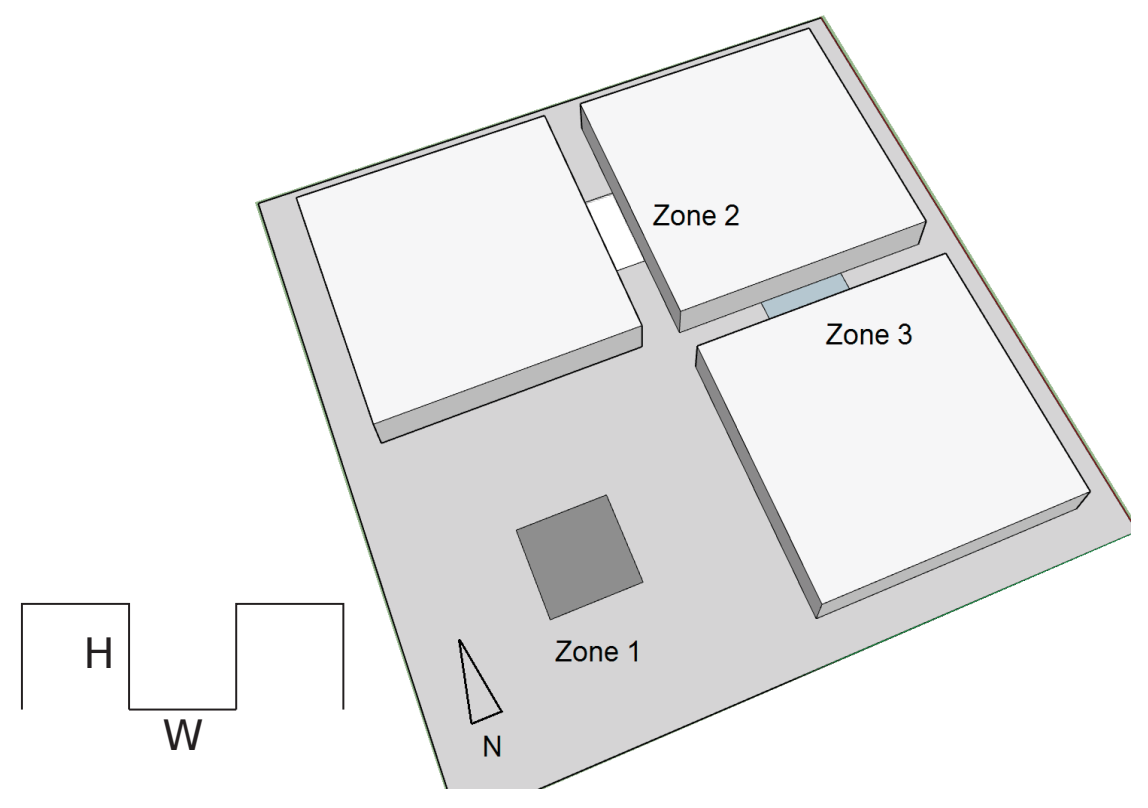

Figure 12: 3D overview of the urban scene

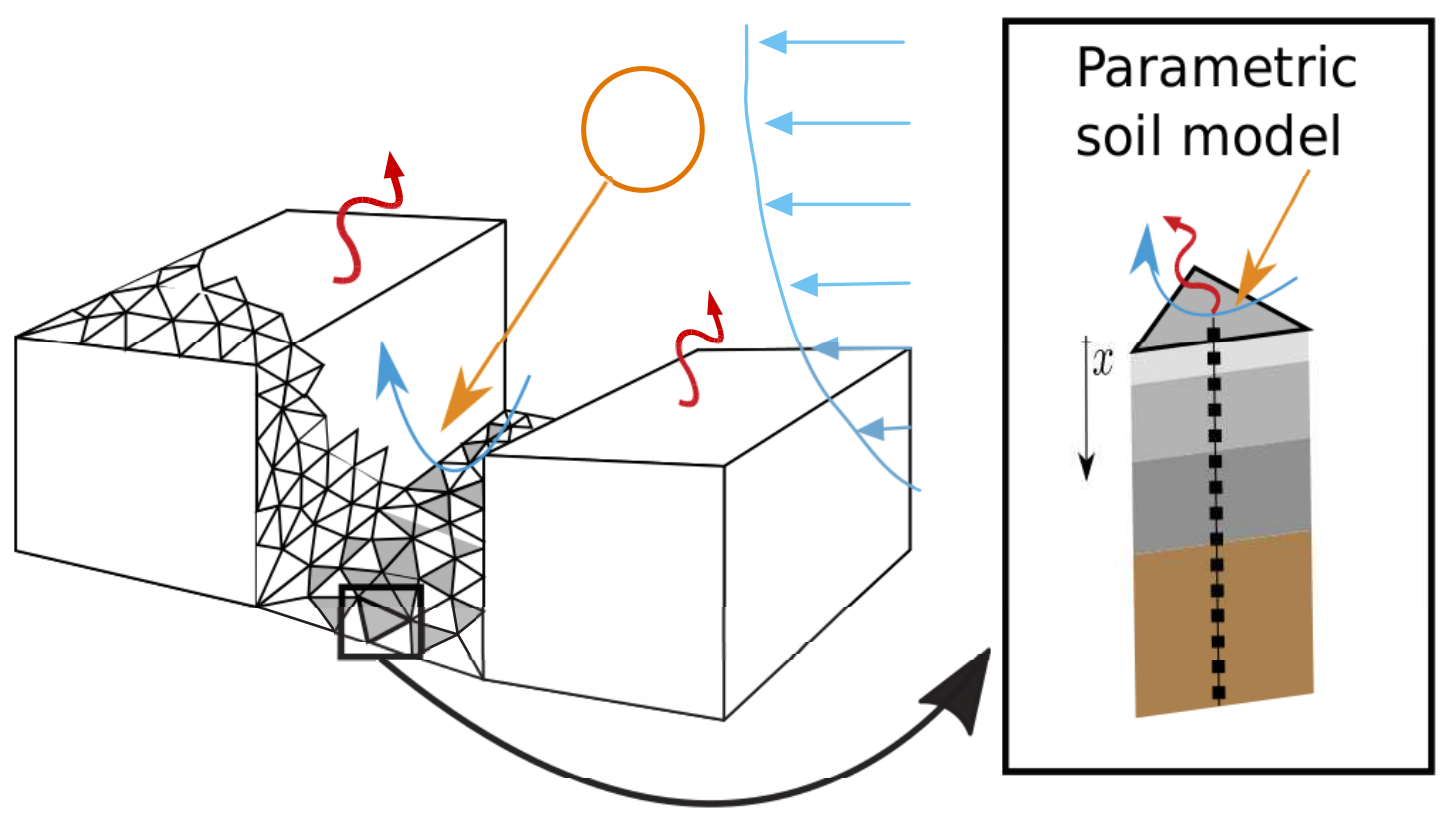

Figure 13: Mesh of the urban geometry. Each surface of the geometry is meshed with triangles, behind which a model has been implemented. In this specific case, the 1D combined parametric soil model is run for each triangle of the floor. For the other triangles (buildings), the 1D SOLENE-microclimat thermal model is employed (see [7] for further details) 


\begin{tabular}{lllll}
\hline $\begin{array}{l}\text { Layer } \\
\text { Number }\end{array}$ & Material & Depth & Thermal conductivity & Volumetric heat capacity \\
\hline 0 & Asphalt & 0.07 & 2.0 & $10^{6} J \cdot m^{-3} \cdot K^{-1}$ \\
1 & Grave & 0.25 & 0.52 & 2.04 \\
2 & Soil & 1.00 & 0.7 & 1.42 \\
\hline
\end{tabular}

Table 3: Thermal characteristics of the soil for the canyon streets case study

and east-west. Figure 12 shows the scene configuration and Figure 13 the mesh, which is built from triangles. The soil represents $32 \%$ of the total mesh content in the urban scene, which comprises 7,372 triangles. The soil is considered to be an impervious urban surface; its thermal properties are listed in Table 3. The soil albedo is 0.10 and its thermal emissivity is 0.95 . The buildings are represented by concrete boxes with $20 \mathrm{~cm}$ of concrete and $10 \mathrm{~cm}$ of external insulation (rock wool). No vegetation has been considered in this case study.

The simulation is run for 6 days in 2010 from April $29^{\text {th }}$ at 7 pm through May $6^{\text {th }}$ at $6 \mathrm{am}$, with a one-hour time step. Only the last two days are used to evaluate the model, while considering the first four days as the necessary model initialization period. Since the SOLENE-microclimat model has been introduced here on a perfectly clear day, a two-day period is sufficient to analyze model behavior under various stresses. Weather data acquired in 2010 for the city of Nantes are used as input model data. These data were recorded by the ONEVU (the IRSTV's Nantes Metropolitan Environmental Observatory, Mestayer et al., 2011 [25]) at the Pin Sec station. Among the observations available, the following data are used as model inputs: air temperature, global and IR radiative fluxes. The combined parametric soil model has been used for each soil triangle in the scene. Three specific zones of this scene, with different net radiative heat flux balances, have been studied:

- The centre of the square (Zone 1 see Figure 12).

- The middle of the north-south canyon street (Zone 2 see Figure 12).

- The middle of the east-west canyon street (Zone 3 see Figure 12). 


\subsubsection{Combined parametric model setup}

In order to model an entire urban scene, the surface heat balance of the combined parametric model needs to be adjusted to take the surrounding surfaces into consideration. For this purpose, the POD+PGD parametric model will be coupled with a microclimate tool. A Robin boundary condition has been considered; it depends on two heat fluxes: a convective heat flux between the surface and the atmosphere, and a net radiative heat flux. The convective flux is calculated with a constant convective heat transfer coefficient: $h=10 \mathrm{~W} \cdot \mathrm{m}^{-2} \cdot \mathrm{K}^{-1}$. The radiative balance between the soil and surrounding surfaces is calculated by the SOLENE-microclimat tool. As noted previously, the problem is first solved by the finite difference method, whereby the finite difference model was coupled with SOLENE-microclimat to simulate a day with a perfectly clear sky. Data from a single day are then used to calculate the POD basis $\Phi$ with five modes $(\mathcal{N}=5)$. The PGD parametric model is built from the initial condition, the net radiative heat flux $q_{\text {net }}$ and the air temperature $T_{a}$ as parameters. This model can be described by the following equation:

$\tilde{T}^{n+1}\left(x, \zeta_{1}, \zeta_{2}, \zeta_{3}, \zeta_{4}, \zeta_{5}, q_{n e t}, T_{a}\right)=\sum_{i=1}^{\mathcal{M}} X_{i}(x) \cdot C_{i}\left(\zeta_{1}\right) \cdot D_{i}\left(\zeta_{2}\right) \cdot E_{i}\left(\zeta_{3}\right) \cdot F_{i}\left(\zeta_{4}\right) \cdot G_{i}\left(\zeta_{5}\right) \cdot H_{i}\left(q_{n e t}\right) \cdot I_{i}\left(T_{a}\right)$.

In the deep soil (below 1 meter), the temperature is assumed constant over the simulation period. Deep in the ground (several meters), the temperature remains constant from one season to the next and can be substituted by the average air temperature [1]. According to this assumption, the depth boundary condition has been set here at the mean air temperature signal over the entire period.

\subsubsection{Coupling method}

As described in the introduction, the SOLENE-microclimat tool is composed of several 1D models assembled with a strong coupling method (i.e. the onion method). It was decided to couple the soil model extracted from the SOLENE-microclimat co-simulation loop. Along these lines, the numerical coupling technique employed was the ping-pong scheme, as described by Hensen (1995 [24]), which represents a weakly coupled strategy. The main idea here is to oscillate between the microclimate model and the parametric soil model. Each model uses the results from the other, as illustrated in Figure 14. First, SOLENE-microclimat 


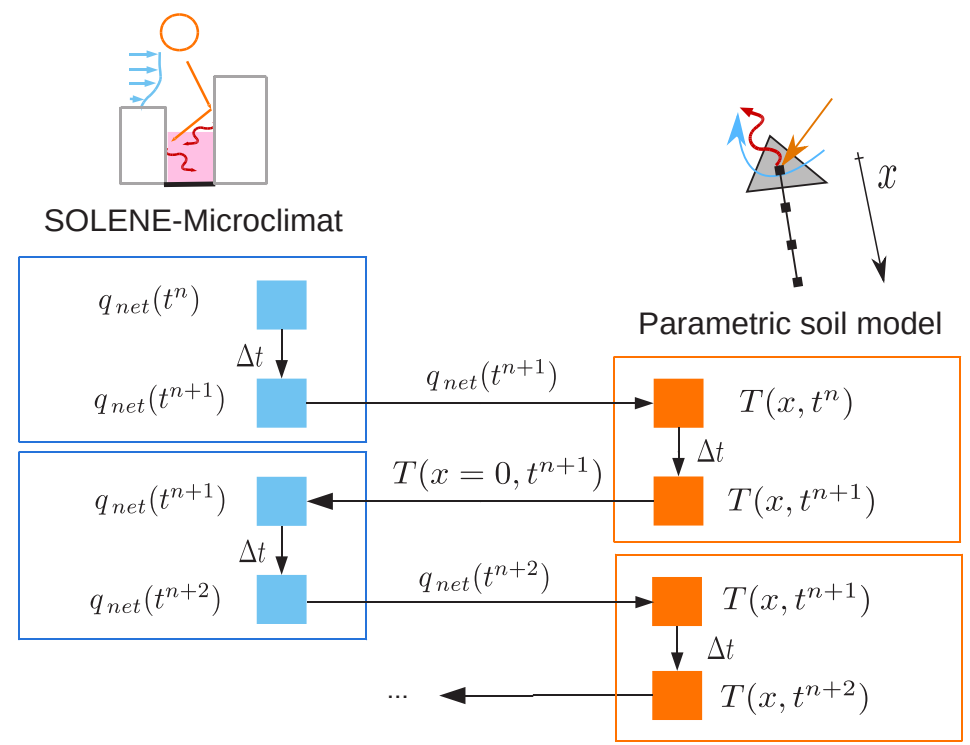

Figure 14: Co-simulation of the parametric soil model with SOLENE-microclimat, the net radiative heat flux $q_{\text {net }}(t)$ and $T(x=0, t)$ as the soil surface temperature

calculates, by means of successive iterations (onion method), the street energy balance (shortand long-wave and convection heat fluxes) until convergence at time step $n+1$. The heat flux balance $\phi_{K, L}(x, n+1)$ is then used by the parametric soil model to calculate the temperature of each surface $T_{\text {soil }}(x, n+1)$. These data are then transferred to SOLENE-microclimat in order to initialize the next time step $(n+2)$ of heat flux balance computation for the time step. The performance of the combined parametric soil model is compared to the finite difference soil model coupled to SOLENE-microclimat in the same manner. The results of the finite difference soil model are adopted as the reference solution. Since the soil models have been extracted from the SOLENE-microclimat co-simulation loop with the ping-pong coupling method, the soil temperature cannot be updated upon each internal SOLENE-microclimat iteration; moreover, the long-wave radiative heat flux cannot be updated with the calculated soil temperature. Such is the main limitation of this coupling method. However, since the coupling limitations are identical for the two outside soils, the error caused by this coupling method on the long-wave radiative heat flux has no impact on the comparative results. 


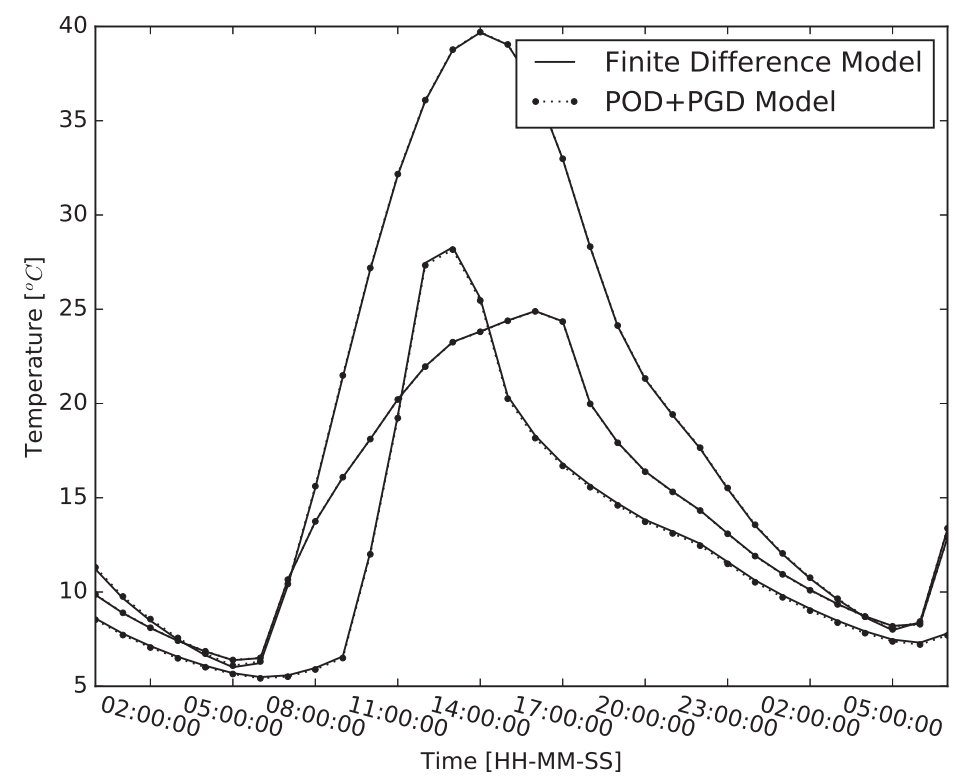

Figure 15: Comparison of the calculated mean surface temperatures over the three zones by both the finite difference and combined parametric soil models. Data from the corresponding zone have been used for the learning step.

\subsubsection{Influence of the learning area}

As discussed above, in order to obtain an efficient reduced-order model, the learning period needs to be as short as possible and representative of soil behavior. In an urban scene, each triangle has a unique surface heat balance that depends not only on meteorological conditions but also on its location (Fig. 13). The location of the triangles used to perform the learning process affects the dynamic model behavior. To evaluate the influence of these conditions on the model, several zones are compared with several learning areas. The aim here is to study the impact of the area assigned to perform the learning step on the results. As indicated in the introduction, surface temperature then becomes the key urban micro-climate variable; it drives the various heat fluxes at the urban surface. In order to evaluate the performance of this reduced model coupled with a microclimate tool, the surface temperature calculated by the combined parametric model is to be compared to that calculated by the finite difference model.

Three learning zones are compared. For each of them, the POD basis is built on the data calculated by the finite difference model for the considered zone. Since the scene is 


\begin{tabular}{llll}
\hline Evaluation zone & Zone 1: & Zone 2: & Zone 3: \\
& the square & North-South street & East-West street \\
Learning zone used & $\varepsilon_{2}(x=0)$ & $\varepsilon_{2}(x=0)$ & $\varepsilon_{2}(x=0)$ \\
Zone 1 & 0.15 & 0.17 & 0.18 \\
\hline Zone 2 & 0.14 & 0.06 & 0.03 \\
\hline Zone 3 & 0.12 & 0.03 & 0.03 \\
\hline \hline
\end{tabular}

Table 4: $\quad \varepsilon_{2}(x=0)\left({ }^{\circ} C\right)$ calculated between the mean temperature output by the finite difference and combined parametric soil models for April $4^{\text {th }}$ and $5^{\text {th }}$ in using different learning zones

\begin{tabular}{llll}
\hline Evaluation zone & Zone 1: & Zone 2: & Zone 3: \\
& the square & North-South street & East-West street \\
Learning zone used & $\varepsilon_{\infty}$ & $\varepsilon_{\infty}$ & $\varepsilon_{\infty}$ \\
Zone 1 & 0.84 & 1.92 & 1.64 \\
\hline Zone 2 & 0.96 & 0.27 & 0.31 \\
\hline Zone 3 & 0.50 & 0.44 & 0.38 \\
\hline \hline
\end{tabular}

Table 5: $\varepsilon_{\infty}\left({ }^{\circ} C\right)$ calculated between the mean temperature output by the finite difference and combined parametric soil models for April $4^{\text {th }}$ and $5^{\text {th }}$ in using different learning zones

composed of thousands of triangles, the mean temperature results over the zone are used to perform the learning step. Figure 15 displays the mean surface temperature calculated over each zone, with a learning stage performed on each corresponding zone. Results are given for a PGD basis with $\mathcal{M}=38$. The mean temperature signals of each zone, calculated with both models, overlap. The ROM (POD+PGD) correctly reproduces the dynamics, with a POD basis of 5 modes.

Lastly, in order to evaluate the behavior of the combined parametric soil model, the $\varepsilon_{\infty}$ error indicator is calculated from the output of the mean temperature of each zone, depending on the learning zone. The $\ell_{2}$ error is also derived at the surface since the surface temperature is the key urban micro-climate variable. Tables 4 and 5 list all these results. To obtain a model as accurate as that presented above at the surface (see Fig. 9), the POD basis comprises 5 modes. For this POD basis and the PGD basis containing 38 modes, the RMSE of the mean surface temperature remains less than $0.20^{\circ} \mathrm{C}$. Depending on the learning zone 


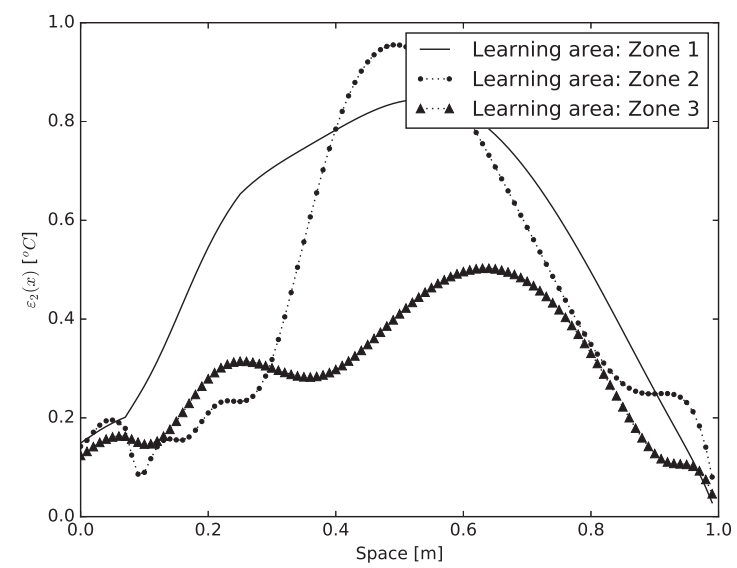

(a) Zone 1

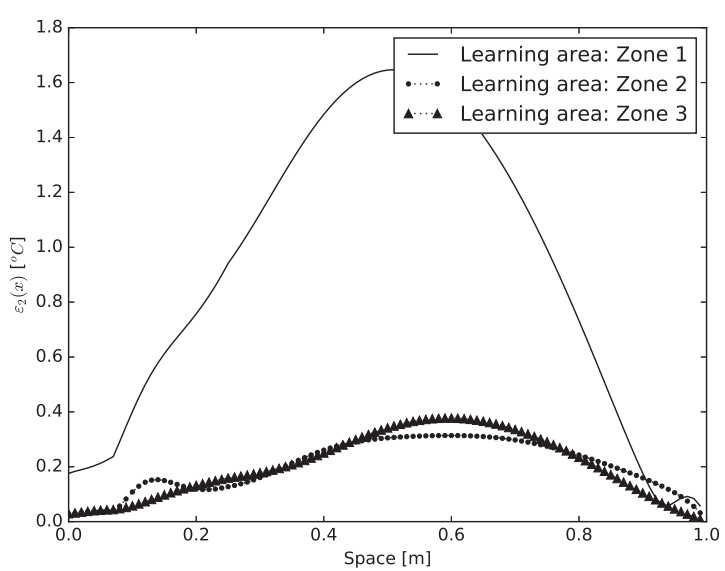

(c) Zone 3 RMSE varies from $0.27^{\circ} \mathrm{C}$ to $1.92^{\circ} \mathrm{C}$.

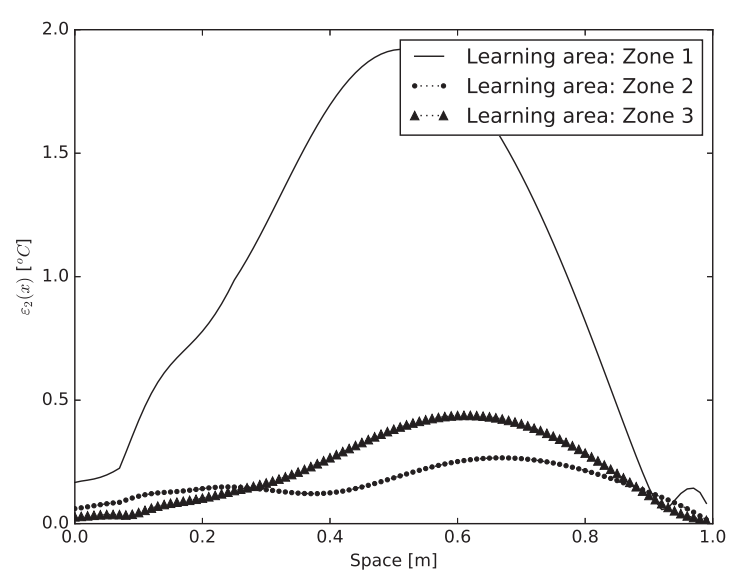

(b) Zone 2

targeted, the loss of accuracy varies from $0.03^{\circ} \mathrm{C}$ to $0.18^{\circ} \mathrm{C}$ at the surface. The maximum 
an amplitude larger than that learned. The two streets (zones 2 and 3) exhibit very different surface heat balances. The temperature signal of zone 2 (Fig. 16(b)) has an amplitude of $27.4^{\circ} \mathrm{C}$. The temperature increases suddenly and then decreases gradually. However, the temperature signal of zone 3 (Fig. 16(c)) gradually increases and then decreases but with a smaller amplitude: $22.2^{\circ} \mathrm{C}$. The dynamic of both temperature profiles is very distinct, which has consequences on the accuracy of the POD+PGD parametric soil model. For each zone, the error is smaller when the learning conditions are met: the calculated area is the same as that used during the learning stage. For the other zones, the loss of accuracy is greater. For example, the maximum Zone 1 error $\varepsilon_{\infty}=0.84^{\circ} \mathrm{C}$ occurs when the learning period is performed on this zone, and $0.1 .92^{\circ} \mathrm{C}$ when the learning process is performed on zone 2 (see Table 5). This finding illustrates that both the learning period and location must be representative of the soil thermal behavior in order to generate accurate results.

\subsubsection{Calculation cost and time}

The model is also evaluated with respect to its computational cost. Two methods are used herein to compare the combined parametric model with the finite difference model, namely the number of problem degrees of freedom, and CPU time. For both the finite difference and combined parametric models, the most expensive operation is to reverse the problem matrix. The number of problem degrees of freedom often yields direct information on the computational complexity of the problem and its cost [16]. With the finite difference model, a system of $\mathrm{N}_{\mathrm{t}}$ time steps and $\mathrm{N}_{\mathrm{x}}$ nodes needs to be solved. With the PGD parametric model, the size of the system depends on $\mathrm{N}_{\mathrm{t}}$ and the number of POD modes $\mathrm{N}$. The computational cost reduction can then be estimated by: $1-\frac{\mathcal{N}}{\mathbb{N}_{t}}$. In our case study, the model was run for 5 modes and 157 time steps (6 days), for a $96.5 \%$ cost reduction.

The previous method however does not take into account all the additional operations, like additions and multiplications, due to the PGD parametric model, the coupling, etc. Consequently, CPU time is used to produce an estimation of the actual drop in computational cost. The CPU times of all simulations (performed on the same computer) were calculated for both soil models (POD+PGD and FD); it includes all online operations due to the model and its coupling with SOLENE-microclimat. The simulation time was averaged over 11 runs 


\begin{tabular}{ll}
\hline Step & CPU time for $\mathcal{M}=38$ \\
\hline Offline no.1: Building the POD basis & 503.5 \\
Offline no.2: Building the PGD parametric model & 375.0 \\
Online : Use of the Finite Difference Model & 503.4 \\
Online : Use of the Combine Parametric model & 99.4 \\
\hline Online Calculation cost reduction & $80 \%$ \\
\hline
\end{tabular}

Table 6: Mean CPU computation time for each step (see Fig. 4 for a description of the offline/online steps)

for each soil model. The simulations performed with the finite difference model required on average $503.4 \mathrm{sec}$, while the combined parametric model time amounted to $99.4 \mathrm{sec}$ with 38 modes. The actual cost reduction for this urban scene is $80 \%$ with 38 modes. The number of modes used in the PGD basis has little influence on the online computation time, yet it does affect the offline steps and the accuracy of the PGD model. This computation time depends on: the urban scene, the duration of the simulated period, the proportion of soil triangles in the scene (32\% in this case), and the specifications of the computer used. However, it does not take into account the time spent offline to build the POD basis.

\section{Conclusion}

The main objective of this paper has been to propose a reduced urban soil model that precisely reproduces the surface temperature and thermal heat flux exchanged between the soil and the urban scene with a reduced computation time. In this aim, a combination of two order-reduction methods has been proposed. The PGD method is used to build a parametric solution, while the POD method configures the initial condition with a minimum number of parameters. The compound method is then composed of an offline phase (learning step plus creation of the parametric model) and an online phase (use of the parametric model).

The POD+PGD parametric soil model was first validated using a simple case with a known analytical solution. The parametric model solutions with 4 modes and the finite difference model produced similar results with an RMSE of $0.03^{\circ} \mathrm{C}$ at the surface and $0.01^{\circ} \mathrm{C}$ at a depth of $0.5 \mathrm{~m}$. This first case study illustrates the numerical behavior of the combined parametric model, which has been considered accurate enough to be applied to other 
situations.

The parametric model was then applied to an actual case study in an open space, more specifically a parking lot. For both models (POD+PGD ROM, FD), the temporal temperature variation at the surface and at several depths was well reproduced compared to the measurements. The loss of accuracy due to model reduction for an entire learning period remains below $0.30^{\circ} \mathrm{C}(\mathrm{POD}+\mathrm{PGD} \mathrm{ROM} / \mathrm{FD})$ at the surface with an RMSE of $1.24^{\circ} \mathrm{C}$ with the measurements (POD+PGD ROM/measurements).

Beyond the loss due to the PGD algorithm, this accuracy drop is due to the POD basis accuracy, which depends directly on the learning period. Various learning periods were compared herein. As expected, the model is accurate when run under the same temporal boundary conditions. Complex signals, such as watering events, are more difficult to model accurately, which is why selecting a short and representative learning period is key to obtaining an efficient parametric model.

The combined parametric soil model was then used in the case of a theoretical urban scene with two canyon streets; it was coupled with the SOLENE-microclimat simulation tool using the ping-pong co-simulation scheme. For a POD basis containing 5 modes and a PGD basis composed of 38 modes, the RMSE of the mean surface temperature remained less than $0.20^{\circ} \mathrm{C}$. Since each triangle of an urban scene has different loadings depending on its location, the accuracy of the model varies with the location of the area used for the learning process.

These two case studies illustrate the difficulties encountered when selecting a representative learning period, which must be short and representative of boundary conditions (meteorological conditions, materials, location of the triangle, etc.) inherent in the simulated soil. The selection of an efficient learning period - sample pair is a new challenge to ensuring model efficiency.

In conclusions, the proposed ROM is able to accurately represent the thermal heat flux exchanged between the soil and the urban scene. For our specific urban case study (30\% of soil triangles in the scene and a one-week simulation period), the computational cost 
reduction amounts to $80 \%$. And this time only takes into account the online part. Further work must now be carried out to improve the offline part, i.e. the learning process.

\section{A. Description of the analytical solution}

The analytical solution $T_{A S}(x, t)$ of problem 1 from the EXACT toolbox [21] is described hereafter.

The problem is normalised with the following dimensionless variables:

$\tilde{x}=\frac{x}{L} ; \tilde{t}=\frac{\alpha t}{L^{2}} ; \tilde{\omega}=\frac{\omega \cdot L^{2}}{\alpha} ; B=\frac{h_{\text {conv }} \cdot L}{k}$

The solution is given by the following equation:

$$
\begin{aligned}
\frac{T_{A S}(x, t)}{T_{\infty}}=\operatorname{Real}\left[B \cdot e^{i \tilde{\omega} \tilde{t}} \cdot \frac{(1+R) \cdot\left[e^{-\tilde{\sigma} \tilde{x}}-e^{-\tilde{\sigma}(2-\tilde{x})}\right]}{2 \cdot \tilde{\sigma}\left(1+R \cdot e^{-2 \tilde{\sigma}}\right)}\right] \\
\\
\quad-2 B \sum_{m=1}^{\infty} \frac{\beta_{m}^{2} \cdot e^{-\beta_{m}^{2} \cdot \tilde{t}}}{\beta_{m}^{4}+\tilde{\omega}^{2}} \frac{\left[\beta_{m} \cos \left(\beta_{m} \tilde{x}\right)+B \sin \left(\beta_{m} \tilde{x}\right)\right] \beta_{m}}{\beta_{m}^{2}+B^{2}+B}
\end{aligned}
$$

with : $\sigma^{2}=\frac{i \omega}{\alpha} ; \tilde{\sigma}=\sigma L ; R=\frac{\tilde{\sigma}-B}{\tilde{\sigma}+B} ;-B=\beta_{m} \cot \beta_{m}$

The notation Real refers to the real part of the complex expression. $\beta_{m}$ values are the positive roots of $\beta_{m} \cot \beta_{m}=-B$.

\section{B. Building the POD basis $\Phi$}

The POD method consists of searching for a set of basis functions $\Phi$ that approximates the temperature profile $T(x, t)$ from the eigenvalues and eigenmodes (Eq. (3)). This method follows three steps:

- First, the LOM is solved. Here, the finite difference model is used to calculate the temperature profile in the soil for a given set of boundary conditions $\left(q_{a}, T_{a}\right)$.

- Then, from this dataset, the reduced-order model is built.

- Finally, this model can be used under a new set of conditions.

The temperature profile obtained from the LOM constitutes a collection of snapshots stored in a matrix $\mathbf{Q}(\mathrm{Nx}, \mathrm{Nt})$, in which each column represents a snapshot of the temperature profile at a given time step. This matrix defines the terms of the learning process and has an impact 
on the reduced model performance. For this reason, the snapshots must be representative of the problem (boundary values, initial conditions, materials used). The basis $\Phi$ capturing the greatest amount of energy from a system with a minimum number of degrees of freedom is formed by the eigenvectors of the problem:

$$
\gamma \cdot \Phi=\lambda . \Phi
$$

$$
\gamma=\mathrm{QQ}^{T}
$$

From the snapshots matrix $\mathbf{Q}$, the auto-correlation matrix $\gamma$ can then be calculated. Next, the eigenmodes (eigenvalues and eigenvectors) are calculated in solving the eigenvalue problem (Equation B.1). The temperature profile is now approximated by $\mathcal{N}$ eigenvectors $\Phi_{i}$ associated with their $\mathcal{N}$ eigenvalues $\lambda_{i}$ with $N_{x} \geqslant \mathcal{N} \geqslant 1$.

$$
\begin{array}{r}
\lambda_{1} \geqslant \ldots \geqslant \lambda_{i} \geqslant \ldots \geqslant \lambda_{\mathcal{N}} \geqslant 0 \\
N_{x} \geqslant \mathcal{N} \geqslant 1
\end{array}
$$

Each eigenvalue represents the portion of energy captured by a mode. Several criteria may be used to select the optimal number of modes. In most examples, an arbitrary threshold is set depending either on the ratio between the first and current eigenvalues $\left(\lambda_{\mathcal{N}}>\eta \lambda_{1}\right)$ or on the portion of energy captured $\left(\frac{\sum_{k}^{\mathcal{N}}=1_{1} \lambda_{k}}{\sum_{i=1}^{N x} \lambda_{i}}>0.999\right)$. In the field of fluid dynamics, Sirovich et al. (1991 [26]) used a combination of both criteria. Moreover, according to Sempey (2007, [27]), these criteria must be adjusted to each specific problem. The reduced basis $\Phi$ of order $\mathcal{N}$ can now be written in the following form:

$$
\Phi=\left(\begin{array}{llll}
\Phi_{1} & \Phi_{2} & \ldots & \Phi_{\mathcal{N}}
\end{array}\right)=\left(\begin{array}{cccc}
\Phi_{1}\left(x_{1}\right) & \Phi_{2}\left(x_{1}\right) & \ldots & \Phi_{\mathcal{N}}\left(x_{1}\right) \\
\Phi_{1}\left(x_{2}\right) & \Phi_{2}\left(x_{2}\right) & \ldots & \Phi_{\mathcal{N}}\left(x_{2}\right) \\
\ldots & \ldots & \ldots & \ldots \\
\Phi_{1}\left(x_{N_{x}}\right) & \Phi_{2}\left(x_{N_{x}}\right) & \ldots & \Phi_{\mathcal{N}}\left(x_{N_{x}}\right)
\end{array}\right)
$$




\section{Development of the alternating direction strategy}

\section{C.1. Computation of $F_{m}^{p}\left(T^{n}\right)$ from $X_{m}^{p}(x)$ and $G_{m}^{p-1}(\psi)$}

Eq. (7) is inserted into Eq. (5), with the following test function (Eq. (C.1)).

$$
u^{*}\left(x, T^{n}, \psi\right)=X_{m}^{p}(x) \cdot F_{m}^{*}\left(T^{n}\right) \cdot G_{m}^{p-1}(\psi)=X \cdot F^{*} \cdot G
$$

We obtain the following equation:

$$
\begin{array}{r}
\int_{\Omega_{x} \times \Omega_{T} \times \Omega_{\psi}} X \cdot F^{*} \cdot G\left(c \frac{X \cdot F \cdot G}{\Delta t}-\frac{\partial}{\partial x}\left(k \frac{\partial X}{\partial x}\right) \cdot F \cdot G\right) \mathrm{d} x \cdot \mathrm{d} T^{n} \cdot \mathrm{d} \psi \\
-\int_{\Omega_{x} \times \Omega_{T} n \times \Omega_{\psi}} X \cdot F^{*} \cdot G \cdot c \frac{T^{n}}{\Delta t} \mathrm{~d} x \cdot \mathrm{d} T^{n} \cdot \mathrm{d} \psi \\
=-\int_{\Omega_{x} \times \Omega_{T} n \times \Omega_{\psi}} X \cdot F^{*} \cdot G \sum_{i=1}^{m-1}\left(c \frac{X_{i} \cdot F_{i} \cdot G_{i}}{\Delta t}-\frac{\partial}{\partial x}\left(k \frac{\partial X_{i}}{\partial x}\right) \cdot F_{i} \cdot G_{i}\right) \mathrm{d} x \cdot \mathrm{d} T^{n} \cdot \mathrm{d} \psi
\end{array}
$$

Since all the functions that depend on parametric coordinate $\psi$ and $x$ are known, they can be integrated over their domain: $\Omega_{x}$ and $\Omega_{\psi}$.

$$
\left\{\begin{array}{rlrl}
x_{1} & = & \int_{\Omega_{x}}(X) \mathrm{d} x \\
x_{2}= & \int_{\Omega_{x}}(X)^{2} \mathrm{~d} x \\
x_{3}= & \int_{\Omega_{x}}\left(X \cdot \frac{\partial}{\partial x}\left(k \frac{\partial X}{\partial x}\right)\right) \mathrm{d} x \\
g_{1}= & \int_{\Omega_{\psi}}(G) \mathrm{d} \psi \\
g_{2}= & \int_{\Omega_{\psi}}(G)^{2} \mathrm{~d} \psi \\
x_{i, 1}= & \int_{\Omega_{x}}\left(X \cdot X_{i}\right) \mathrm{d} x \\
x_{i, 2}= & \int_{\Omega_{x}}\left(X \cdot \frac{\partial}{\partial x}\left(k \frac{\partial X_{i}}{\partial x}\right)\right) \mathrm{d} x \\
g_{i}= & \int_{\Omega_{\psi}}\left(G \cdot G_{i}\right) \mathrm{d} \psi
\end{array}\right.
$$

${ }_{642}$ We derive the following algebraic equation (12), whose direct solution yields the function F.

$$
F \cdot\left(\frac{c}{\Delta t} x_{2} g_{2}-x_{3} g_{2}\right)=-\sum_{i=1}^{m-1}\left(c \frac{F_{i}}{\Delta t} x_{i, 1} g_{i}-F_{i} x_{i, 2} g_{i}\right)+x_{1} g_{1} c \frac{T^{n}}{\Delta t}
$$

\section{C.2. Computation of $G_{m}^{p}(\psi)$ from $X_{m}^{p}(x)$ and $F_{m}^{p}\left(T^{n}\right)$}

Eq. (7) is inserted into Eq. (5), with the following test function (Eq. (C.4))

$$
u^{*}\left(x, T^{n}, \psi\right)=X_{m}^{p}(x) \cdot F_{m}\left(T^{n}\right) \cdot G_{m}^{*}(\psi)=X \cdot F \cdot G^{*}
$$




$$
\begin{array}{r}
\int_{\Omega_{x} \times \Omega_{T} n \times \Omega_{\psi}} X \cdot F \cdot G^{*}\left(c \frac{X \cdot F \cdot G}{\Delta t}-\frac{\partial}{\partial x}\left(k \frac{\partial X}{\partial x}\right) \cdot F \cdot G\right) \mathrm{d} x \cdot \mathrm{d} T^{n} \cdot \mathrm{d} \psi \\
-\int_{\Omega_{x} \times \Omega_{T} n \times \Omega_{\psi}} X \cdot F \cdot G^{*} \cdot c \frac{T^{n}}{\Delta t} \mathrm{~d} x \cdot \mathrm{d} T^{n} \cdot \mathrm{d} \psi \\
=-\int_{\Omega_{x} \times \Omega_{T} n \times \Omega_{\psi}} X \cdot F \cdot G^{*} \sum_{i=1}^{m-1}\left(c \frac{X_{i} \cdot F_{i} \cdot G_{i}}{\Delta t}-\frac{\partial}{\partial x}\left(k \frac{\partial X_{i}}{\partial x}\right) \cdot F_{i} \cdot G_{i}\right) \mathrm{d} x \cdot \mathrm{d} T^{n} \cdot \mathrm{d} \psi
\end{array}
$$

We obtain the following equation:

Since all the functions that depend on parametric coordinate $x$ and $T^{n}$ are known, they can be integrated over their domain: $\Omega_{x}$ and $\Omega_{T^{n}}$.

$$
\left\{\begin{array}{rrr}
x_{1}= & \int_{\Omega_{x}}(X) \mathrm{d} x \\
x_{2}= & \int_{\Omega_{x}}(X)^{2} \mathrm{~d} x \\
x_{3}= & \int_{\Omega_{x}}\left(X \cdot \frac{\partial}{\partial x}\left(k \frac{\partial X}{\partial x}\right)\right) \mathrm{d} x \\
f_{1}= & \int_{\Omega_{T^{n}}}(F) \mathrm{d} T^{n} \\
f_{2}= & \int_{\Omega_{T^{n}}}(F)^{2} \mathrm{~d} T^{n} \\
x_{i, 1}= & \int_{\Omega_{x}}\left(X \cdot X_{i}\right) \mathrm{d} x \\
x_{i, 2}= & \int_{\Omega_{x}}\left(X \cdot \frac{\partial}{\partial x}\left(k \frac{\partial X_{i}}{\partial x}\right)\right) \mathrm{d} x \\
f_{i} & = & \int_{\Omega_{T^{n}}}\left(F . F_{i}\right) \mathrm{d} T^{n}
\end{array}\right.
$$

We derive the following algebraic equation (13), whose direct solution yields the function G.

$$
G\left(\frac{c}{\Delta t} x_{2} f_{2}-x_{3} f_{2}\right)=-\sum_{i=1}^{m-1}\left(c \frac{G_{i}}{\Delta t} \cdot x_{i, 1} \cdot f_{i}-G_{i} x_{i, 2} f_{i}\right)+x_{1} f_{1} c \frac{T^{n}}{\Delta t}
$$

\section{References}

[1] T. R. Oke, Boundary layer climates, Routledge, 2002.

[2] T. Oke, G. Johnson, D. Steyn, I. Watson, Simulation of surface urban heat islands under 'ideal'conditions at night part 2: Diagnosis of causation, Boundary-Layer Meteorology 56 (1991) 339-358.

[3] G. Johnson, T. Oke, T. Lyons, D. Steyn, I. Watson, J. A. Voogt, Simulation of surface urban heat islands under 'ideal'conditions at night part 1: Theory and tests against field data, Boundary-Layer Meteorology 56 (1991) 275-294. 
[4] V. Masson, A physically-based scheme for the urban energy budget in atmospheric models, Boundary-layer meteorology 94 (2000) 357-397.

[5] R. Tavares, I. Calmet, S. Dupont, Modelling the impact of green infrastructures on local microclimate within an idealized homogeneous urban canopy, in: ICUC9-9th International Conference on Urban Climate jointly with 12th Symposium on the Urban Environment Modelling, pp. 1-6.

[6] J. Bouyer, Modelisation et simulation des microclimats urbains-Etude de l'impact de l'amenagement urbain sur les consommations energetiques des batiments, Ph.D. thesis, Universite de Nantes, 2009.

[7] M. Musy, L. Malys, B. Morille, C. Inard, The use of solene-microclimat model to assess adaptation strategies at the district scale, Urban Climate 14 (2015) 213-223.

[8] X. Yang, L. Zhao, M. Bruse, Q. Meng, Evaluation of a microclimate model for predicting the thermal behavior of different ground surfaces, Building and Environment 60 (2013) 93-104.

[9] A. Gros, Modélisation de la demande énergétique des bâtiments à l'échelle d'un quartier, Ph.D. thesis, Université de La Rochelle, 2013.

[10] M.-H. Azam, B. Morille, J. Bernard, M. Musy, F. Rodriguez, A new urban soil model for solene-microclimat: Review, sensitivity analysis and validation on a car park, Urban Climate (2017) in press.

[11] S. Gasparin, M. Chhay, J. Berger, N. Mendes, A hybrid analytical-numerical method for computing coupled temperature and moisture content fields in porous soils, Journal of Building Physics (2017) 1744259117720644.

[12] D. González, F. Masson, F. Poulhaon, A. Leygue, E. Cueto, F. Chinesta, Proper generalized decomposition based dynamic data driven inverse identification, Mathematics and Computers in Simulation 82 (2012) 1677-1695. 
[13] J. Berger, S. Gasparin, M. Chhay, N. Mendes, Estimation of temperature-dependent thermal conductivity using proper generalised decomposition for building energy management, Journal of Building Physics 40 (2016) 235-262.

[14] J. Berger, N. Mendes, An innovative method for the design of high energy performance building envelopes, Applied Energy 190 (2017) 266-277.

[15] J. Berger, S. Guernouti, M. Woloszyn, F. Chinesta, Proper generalised decomposition for heat and moisture multizone modelling, Energy and Buildings 105 (2015) 334-351.

[16] J. Berger, W. Mazuroski, N. Mendes, S. Guernouti, M. Woloszyn, 2d whole-building hygrothermal simulation analysis based on a pgd reduced order model, Energy and Buildings 112 (2016) 49-61.

[17] F. Poulhaon, F. Chinesta, A. Leygue, A first step toward a pgd-based time parallelisation strategy, European Journal of Computational Mechanics/Revue Européenne de Mécanique Numérique 21 (2012) 300-311.

[18] E. Cueto, D. González, I. Alfaro, Proper Generalized Decompositions, SpringerBriefs in Applied Sciences and Technology, Springer International Publishing, Cham, 2016. DOI: 10.1007/978-3-319-29994-5.

[19] F. Chinesta, R. Keunings, A. Leygue, The proper generalized decomposition for advanced numerical simulations: a primer, Springer Science \& Business Media, 2013.

[20] D. González, E. Cueto, F. Chinesta, Real-time direct integration of reduced solid dynamics equations, International Journal for Numerical Methods in Engineering 99 (2014) 633-653.

[21] K. D. Cole, J. Krahn, Analytical solution x31b60t0, slab body with cosine-periodic fluid convection at $\mathrm{x}=0$ and zero temperature at $\mathrm{x}=\mathrm{l}$, Exact analytical conduction toolbox (2015).

[22] J. Cohard, J. Rosant, F. Rodriguez, H. Andrieu, P. Mestayer, P. Guillevic, Energy and water budgets of asphalt concrete pavement under simulated rain events, Urban Climate (2017) in press. 
[23] M.-H. Azam, J. Bernard, B. Morille, M. Musy, H. Andrieu, A pavement-watering thermal model for solene-microclimat: Development and evaluation, Urban Climate 25 (2018) 22-36.

[24] J. Hensen, Modelling coupled heat and airflow: ping pong vs. onions, in: DOCUMENTAIR INFILTRATION CENTRE AIC PROC, OSCAR FABER PLC, pp. 253-253.

[25] P. Mestayer, J.-M. Rosant, F. Rodriguez, J.-M. Rouaud, La campagne expérimentale fluxsap 2010: Mesures de climatologie en zone urbaine hétérogène (2011).

[26] L. Sirovich, A. E. Deane, A computational study of rayleigh-bénard convection. part 2. dimension considerations, Journal of fluid mechanics 222 (1991) 251-265.

[27] A. Sempey, Prise en compte du champ thermo-convectif pour le contrôle thermique des espaces habitables, Ph.D. thesis, Université de La Rochelle, 2007. 